\title{
VIII.
}

\section{Ueber Thermoneurosen.}

(Material zur Pathologie der vasomotorischen Neurosen.)

Fon Dr. M. M. Scherschewsky in St. Petersburg.

\footnotetext{
„Fin Zuviel von Thatsachen ist noch niemals bedenklich, ein Zuwenig oft genug gefährlich geworden."

Heidenhain (Pflüger's Arch, 1878. s.31).
}

Trotz vielfacher Untersuchungen über diejenige Rolle, welche das Nervensystem im Fieberprozess spielt, bleibt diese Frage auch jetzt noch unentschieden. Das grosse Interesse, welches die Forschung in diesem äusserst wichtigen Abschnitte der Pathologie darbietet, hat sowohl früher, als auch jetzt, jeden Versuch gerechtfertigt, wenn auch indirect diese höchst wichtige Frage, wenn nicht zu lösen, so doch einigermaassen zu erläutern. Der Klinik fällt auch in diesem Falle, wie uns scheint, eine recht bedeutende Rolle zu. Da sie im Besitze eines ungemein grossen Vorrathes individueller Formen ist, muss sie beim Studium der Einzelheiten nicht nur die schon gewonnenen physiologischen Gesetze in jedem gegebenen Falle anwenden, sondern auch vielleicht neue Gesetze auffinden. Eine solche Aufgabe erfordert natürlich eine Masse von Material und Beobachtungen. In Anbetreff dieses Umstandes, erlauben wir uns einige uns zur Verfïgung stehende Fälle den Collegen mitzutheilen. Wir haben die Fälle in den Jahren 1875 bis 1883 beobachtet und veröffentlichen sie in der Form und Reihenfolge, in welcher wir sie notirt haben, mit all' den Fehlern und Lücken, welche theils durch die Lebensweise der Kranken, theils aber auch durch die Mängel der Beobachtung selbst bedingt sind.

Fall I. F. L., 12 Jahre, die älteste Tochter einer mit 7 Kindern gesegneten unbemittelten Kaufmannsfamilie erkrankte am 1.Januar 1875 an Halsschmerzen und Sehlingbeschwerden mit ausgesprochenem allgemeinem Unbehagen. Die Kranke haben wir am Abend des ersten Tages gesehen. Pat. ist von gutem Wuchs, mager, soll seit der Kindheit immer kränklich gewesen sein, an einer ernsten Krankheit aber nie gelitten haben. 
Die Untersuchung ergab einen ausgebreiteten diphtheritischen Belag auf den beiden Tonsillen, aw Gaumenbogen und an der Uvula. Abendtemperatur 39,4. Puls 100, frequent, klein, jedoch regelmässig.

Am 6. Januar war der Rachen vollständig rein, die Temperatur normal; es stellte sich Appetit ein. Zu dieser Zeit erkrankten alle übrigen Familienglieder an Diphtherie, da aus verschiedenen Crsachen das Isoliren der Patientin unmöglich war.

Bis zum 15. Januar blieb die Kranke scheinbar vollkommen gesund. In der Nacht vom 15. auf den 16. zeigten sich die ersten Symptome einer Larynxdiphtherie, welche im Verlauf von 24 Stunden rapid ihr Maximum erreichte. Die Athembeschwerden und Cyanose nahmen überhand, so dass am 19., da Erstickungsgefahr drohte, bereits alle Vorbereitungen zu einer Tracheotomie getroffen waren. Die einzelnen Erscheinungen des klinischen Bildes bei Seite lassend, da sie für unsere Zwecke keine Bedeutung haben, erwähnen wir nur noch, dass wir uns am Abend des 19. - bevor wir zur Operation schritten - für ein heisses Dampf bad entschieden. Pat. wurde in warme Decken gehüllt - auf 10 Minuten zwisehen 2 grosse, offen gebaltene, kochende Theemaschinen gesetzt. Nach einer Pause von 10 Mínuten wurde dieses improvisirte Dampf bad nochmals wiederholt, da der erste Versuch das Athmen bedeutend exleichtert hatte, obgleich nur auf kurze Zeit. Das zweite Dampfbad dauerte circa 20 Minuten; nach 10--12 Minuten stellte sich ein heftiger Hustenanfall ein, welcher 3-4 Minuten anhielt und mit starkem Erbrechen endete, wobei eine sehr grosse Menge von zum Theil sich verästelnden Membranen ausgebrochen wurde. Sofort stellte sich eine merkliche Erleichterung ein; Cyanose verschwand und die Operation konnte auf diese Weise umgangen werden.

20. Jan. Allgemeinbefinden gut; Athem frei; die Ternperatur, welche während der zweiten Erkrankung zwischen 38,5 und $39,8^{\circ}$ schwankte, sank am Morgen bis $37,6^{\circ}$. Die allgemeine Besserung schritt fort und bis zum 24. Jan. waren alle krankhaften Erscheinungen vollständig geschwunden; Morgentemperatur $36,4-36,8^{\circ}$, Abendtemperatur $37,1-37,3^{\circ}$. Die Kräfte nahmen zu, so dass gegen den 1. Februar keine Spur von Krankheitserscheinungen vorhanden war. In Anbetracht der möglicher Weise nachfolgenden Lähmungen blieb Pat. noch unter weiterer Beobachtung.

Am Morgen des 16. Februar haben wir zuerst einen kaum bemerkbaren Strabismus constatiren können; schon gegen Abend stellte sich eine näselnde Stimme, erschwerte Aussprache und Ausfliessen der zu sich genommenen Flüssigkeit durch die Nase ein; die Kranke ist nicht im Stande, die Wangen aufzublasen oder ein licht auszulöschen. Bei offenem Munde und ausgestreckter Zunge sieht man, dass das Gaumensegel unbeweglich bleibt und die Zungenwurzel die Uvula berührt. Die Berührung dieser Theile ist unempfindlich. Die Herzthätigkeit ist sehr stürmisch, die Herzschläge sind ziemlich schwach, aber frequent; die Herztöne rein; Puls 120; Temp. 36,90.

17. Febr. Pat. ist nicht im Stande zu schlucken, flüssige Nahrung fliesst durch die Nase zurück, feste Nahrung gelangt in den Kehlkopf und ruft 


\section{3}

starken Husten uid drohende Athembeschwerden hervor. Herzklopfen sehr stark. Strabismus mehr ausgesprochen. Klagen über Ameisenkriechen in den Händen und grosse Schwäche in den Füssen. Der Gang schwankend. Die Hautempindlichkeit an den Füssen geschwächt. Temperatur am Morgen $37,0^{0}$, am Abend $37,3^{\circ}$.

Am 18. Fębr.: Ausgesprochene Lähmungserscheinungen im Rachen. Das Schlucken ganz unmöglich. Nährklystiere verordnet. Temp. 39,90.

Die beiliegende Curve zeigt den Temperaturverlauf vom 17. Februar bis 2. März. Die Lähmungserscheinungen während dieser Periode blieben in statu quo. Die wiederholte Harnuntersuchung fiel negativ aus.

Fig. 1 .

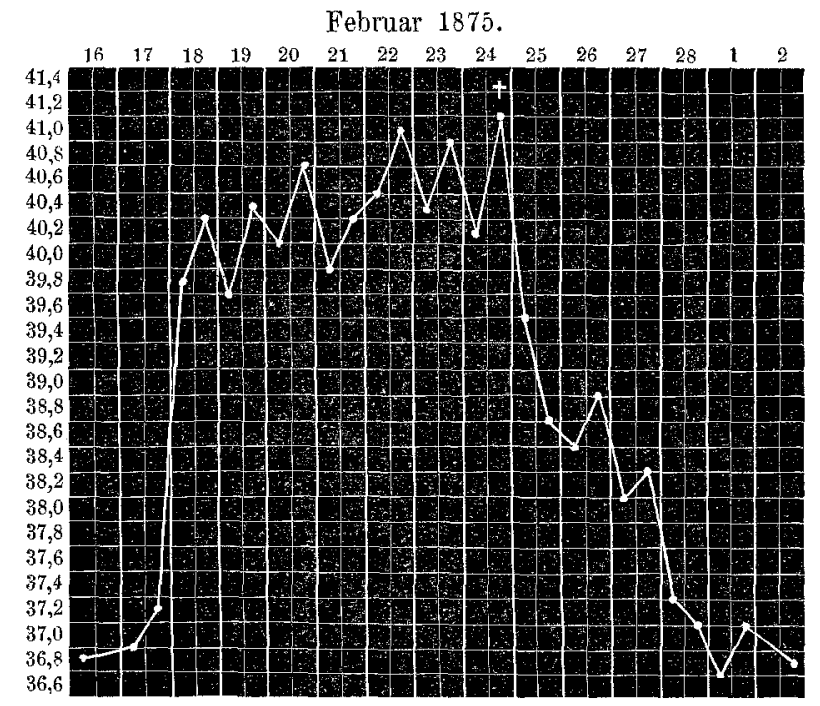

Am Abend des 18. stieg die Temperatur bis 40,40. Ungeachtet einer so hohen Temperatur fühlt sich die Haut fast normal an, ist blass und trocken. Der Puls ist klein, fadenförmig, frequent, 120. Die Respiration kurz, beschleunigt, kein bedeutender, aber lästiger, trockener, scharfer Husten.

Der weitere Krankheitsverlauf zeigte ganz unbedeutende Schwankungen, daher um Wiederholungen za vermeiden, sei noch bemerkt, dass bei einer so hohen Temperatur wir unser Bestreben dahin lenkten, dieselbe um jeden Preis herabzusetzen. Da wir zu jener Zeit nicht im Stande waren eine genügende Erklärung dieser hohen Temperatur aufzufinden, brachten wir selbstverständlich rein empirische antipyretische Mittel in Anwendung. Aber auch die consequente Anwendung derselben, sogar in grossen Gaben, brachte gar keine Resultate mit sich; und noch am Morgen des 24. Februar blieb die Temperatur auf $40,3^{\circ}$, am Abend $41,2^{\circ}$. 
Die Sehwäche der Pat. nahı zu dieser Zeit einen sehr drohenden Charakter an, obgleich die Nährklystiere ununterbrochen fortgesetzt wurden; die Erregtheit des Herzens gab den gewöhulichen Mitteln nicht nach; es stellten sich Herzintermittens und Arythmie ein. In Anbetracht der Erfolglosigkeit unserer Therapie verordneten wir am Abend des 24. forcirtes Einathmen von Sauerstoff, theils um die verlöschenden Lebenskräfte zu unterhalten, theils unter dem Einflusse der damals herrschenden Vorliebe für den Gebrauch dieses Mittels. Wir motivirten damals einigermaassen unsere Verordnung durch starke Anämie und besonders durch den sehr anämischen Zustand des Gehirns.

Das Resultat der Verordnung des Sauerstoffs ist aus der Curve ersicbtbar - zum 28. fiel die Temperatur bis zur Norm.

Der weitere Krankheitsverlauf bietet nichts Besonderes dar - die Kranke erholte sich nur langsam, bei fortdauerndem Gebranch von Sauerstoff; um den 20. März verschwanden alle paralytischen Erscheinungen.

Dies ist der Verlauf des Falles, welcher bis auf die Temperaturerhöhung nach dem Eintritt der paralytischen Erscheinungen gar keine Abweichungen vom fast classischen Typus der Diphtheritis mit consecutiven Paralysen darbot. Das ganze Interesse und die Eigenthümlichkeit desselben liegen in der hohen Temperatur, welche die paralytischen Erscheinungen begleitete. Für uns persönlich war es nicht minder interessant die Rolle des Sauerstoffs im gegebenen Falle zu erklären, - mit anderen Worten, zu entscheiden, ob die Temperaturerniedrigung wirklich die Folge des Sauerstoffgebrauchs oder eine zufällige Erscheinung war. Diese Frage hätte man wohl durch einen Controlversuch, mit temporärem Weglassen des Sauerstoffs, entscheiden können; ein solches Experiment wäre jedoch bei dem gefährlichen Zustande der Kranken kaum erlaubt gewesen, um so mehr, da das sichtbare Resultat des Sauerstoffgebrauchs so schnell eintrat. Wie dem auch sei, sogar wenn wir zugeben, dass der Sauerstoff die Temperaturerniedrigung bewirkt habe, so bleibt uns doch das Wesen der Frage, d. i. die Thatsache der deutlichen Temperaturerhöhung . ungelöst. Wir haben die reiche Literatur der Diphtheritis durchgesehen, so weit es uns damals möglich war, doch sind wir keinem einzigen Falle begegnet, der in thermischer Beziehung Aehnlichkeit mit dem unsrigen hätte. Deshalb mussten wir uns mit der Voraussetzung begnügen, dass unser Fall eine „individuelle Eigenthümlichkeit" der genannten Kranken bildete.

Den nächsten Fall sahen wir Ende 1876. 
Fall II. 9jähriges Mädchen, das einzige Kind sehr intelligenter Eltern. Die Mutter litt bis zu ibrer Verheirathing an stark ausgeprägter Neurasthenie, complicirt durch Darmneurose; nach der Geburt dieses Kindes erkrankte sie an Graves'scher Krankheit. Der Vater, nervös im allgemeinsten Sinne dieses Wortes, leidet oft an starker Migräne; er beschäftigt sich ansschliesslich mit geistigen Arbeiten.

Dieses Kind kennen wir seit seiner Geburt und die folgenden Daten sind theils von uns selbst notirt, theils aus dem exemplarisch geführten Journal der Mutter geschöpft.

Das Kind wurde in Folge ungünstiger Umstände mit Kuhmilch aufgezogen; im 3,-4. Lebensmonate machten sich die Symptome einer entstehenden Rachitis bemerkbar. Es stellten sich Kopfschweisse, Zuckungen, Aufschreien im Schlafe, Krämpfe in den Extremitäten und ein scheinbar grundloses Schreien ein, welches zuweilen stundenlang anhielt. Während der Anwendung einer entsprechenden Cur verschwanden die Symptome der Rachitis sehr bald; die nervösen Erscheinungen jedoch dauerten fort. $\mathrm{Zu}$ den letzteren musste nach einer langen Beobachtungszeit auch jenes Schreien gezäblt werden, was auch durch den Umstand bestätigt wurde, dass das Schreien bei alleiniger Anwendung yon Bromkalium nachliess. So machte sich denn schon fräh der Einfluss der Nervosität geltend und bereits im 8. Lebensmonat musste zu den Antinervina Zuflucht genommen werden, obgleich das Kind gut genährt war und mit 12 Monaten das äussere Bild blühender Gesundheit darbot. Gegen Ende des ersten Lebensjahres verschwinden obengenannte Symptome aImählich, um einem neven Platz ur machen. Von Zeit zu Zeit stells sich, ohne irgend welche wahrnehmbare Ursachen, ein Fieberzustand ein, die Temperatur steigt plötzlich bis $38^{\circ}$ mit einigen Zehnteln und erreicht in $2-3$ Stunden rasch $40^{\circ}$; sie bält sich auf dieser Höhe 5-7 Stunden und zeigt zuweilen $40,8^{\circ}$, um darauf raseh bis zur normalen Höhe herabzusinken. Gleichzeitig mit der Temperaturerhöhung stellte sich Salivation (klebriger, fadenziehender Speichel) und ein geringes Aufgetriebensein der Magengegend ein. Stuhlgang hat die Kranke dabei zwar täglich, doch war die Defäcation an Quantität ungenügend. Zuweilen, was indessen seltener vorkam, war die Temperaturerhähung sogar von unwillkürlichen flüssigen Stühlen begleitet. Solche Fieberparoxysmen wiederholten sich einigemal im Jahre und waren stets mit heftigem Herzklopfen verbunden, wobei der Pulsschlag bis 150 stieg und, was besonders betont werden muss, die Haut sich kühl anföhlte. Dieser Zustand der Hautdecken kam auch bei allon folgenden Paroxysmen zur Beobachtung und war nicht selten Ursache einer zu späten Temperaturmessung. Kopfschmerz und Lichtscheu wurden nie beobachtet.

Mit dem Wachsthum des Kindes nabm auch die Nervosität zu. Lis entwickelte sich nicht dem Alter gemäss und früh schon stellten sich eine seltene Eigenliebe und Empfindlichkeit ein - ein unvorsichtiges Wort des leidenschaftlich geliebten Vaters war im Stande sofort Thränen und Temperaturerhöhung, die durch das Thermometer controlirt wurde, hervorzurufen. 
Im 4. Lebensjahre wiederholte sich dieser Zustand wieder einmal. Nachdem der Appetit 2-3 Tage lang sehr launisch gewesen, hob sich die Temperatur bis $38^{\circ}$, bei kleinem, frequentem Puls, $140-150$ und starker Salivation. Dieser Zustand dauerte 2 Wochen, wobei die Temperatur an ein und demselben Tage zwischen $36,4-38,8^{0}$ schwankte. Dabei stellte sich ab und zu heftiges Erbrechen ein, welches nur dureh horizontale Lage mit gesenktem Kopfe beseitigt werden konnte. Das Sensorium war während dieser ganzen Zeit frei, das Kind spielte, jedoch nur auf dem Rücken liegend. Da sich in den Organen keinerlei Veränderungen auffinden liessen und alle gebräucblichen Maassregeln erfolglos angewandt worden, nahm ich meine Zuflucht zum Bromkalium und verordnete ausserdem Charnpagner, da Zeichen einer Hirnanämie vorhanden waren. Während des Gebrauches dieser, Mittel stellte sich fester Schlaf ein und am Morgen war das Kind gesund.

- Solche Anfälle, jedoch von kurzer Dauer, wiederholten sich bis zum Alter von 4 Jahren 8 Monaten noch einige Male, wobei die Temperatur bis 40,8 $8^{\circ}$ stieg und jedesmal Salivation, selten aber Erbrechen eintrat. Während derselben blieb das Kind meist auf den Füssen. Das Bromkalium bewirkte auch jetzt rasche Besserung.

Der folgende Paroxysmus trat im 5. Lebensjahre auf. Sein Charakter blieb derselbe, es stellte sich jedoch hier zuerst Arythmie in Gestalt eines bald interwittirenden, bald arythmischen Pulses ein.

Fig. 2. Januar 1881.

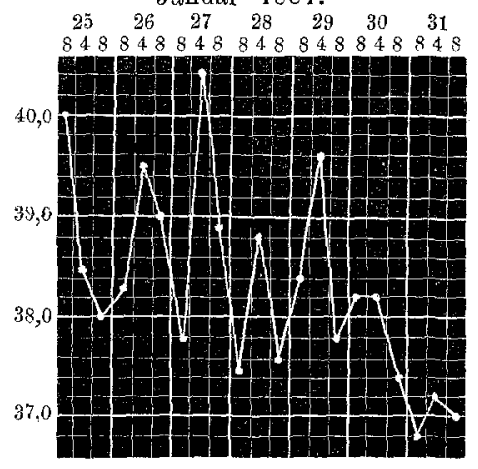

Als das Kind 6 Jahre 2 Mon. alt war, stieg nach einer starken geistigen Aufregung (Trennung rom Vater) die Temperatur plötzlich bis $40^{\circ}$ mit bedeutendem Schüttelfrost; dabei starkes Herzklopfen mit Arythmie. Dieser Zustand dauerte 6 Tage, wie aus der beifolgenden Figur ersehen 'werden kann, Puls 120-150. Während dieses Anfalles offenbarte sich deutlich der krampfhafte Zustand der Gedärme; zuerst Obstipation, darauf 2 unwillkürliche (jedoch compacte) Stuhlgänge. Ausserdem konnten hier zuerst profuse Schweisse constatirt werden, an welche jedoch niemals Temperaturerniedrigung sich anschloss; der Puls wurde unregelmässig, die Arythmie deutlicher.

Zwei Monate später stellte sich, nach einer Kränkung, ein neuer Anfall ein, Temperatur $40,2^{\circ}$, Puls 140 . Uebelkeit und Salivation fehlten und es wurde Chinin verordnet, in der Voraussetzung, dass dies der Anfang irgend einer allgemeinen Krankheit sei; die Temperatur wurde jedoch in keinerlei Weise vom Chinin beeinflusst. Diesmal bewirkte auch das Bromkalium nur eine geringe Temperaturherabsetzung. Da gleichzeitig das Abdomen stark aufgetrieben war, so lag der Gedanke an die Möglichkeit eines Spasmus der 
Gedärme nabe, es wurde daher Belladonna verordnet. Einige Stunden nach der ersten Gabe fing die Temperatur an rasch zu fallen, nach der zweiten Gabe sank sie zur Norm herab.

Diese Complication der Anfälle durch Intestinalspasmus wiederholt sich in der Folge sehr oft; jedesmal erwiesen sich Belladonna und Bromkalium sehr wirksam dagegen, wobei auch zugleich das Fieber nachliess.

Vom 7. Lebensjahre an werden die Anfälle seltener und schwächer: die Temperatur steigt selten äber $39^{\circ}$, wie es aus einer der vielen späteren Curven zu ersehen ist, dafür aber prägen sich die ursächlichen Momente der Anfälle um so deutlicher aus. Ein unangenehmer Traum, irgend ein Lärm, sogar das Erscheinen eines fremden Menschen im Hause genügen, um einen Anfall hervorzurufen. Ias Besuchen des Theaters hatte immer einen 2-3tägigen Anfall zur Folge.

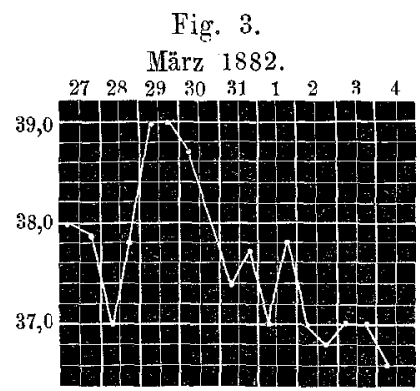

Die in den freien Intervallen zu verschiedenen Zeiten vorgenommene Untersuchung gab keinerlei Anhaltspunkte. Das nervöse Kind fällt durch seine frühzeitige Entwicklung und Urtheilskraft, durch seine Gutmüthigkeit, Anbänglichkeit und Reizbarkeit auf. Dabei ist es sonst gesund und äussert keinerlei Klagen. Das Herz ist normal, die Töne rein, keine Geräusche vorhanden. Der Puls gewöhnlich 80. Das Kind geht frei und leicht, ohne dass sich die geringste Athemnoth einstellt oder die Herzthätigkeit bedentend beschleunigt wird. Nach anhaltendem Laufen beschleunigt sich der Pulsschlag kaum um 10-15 Schläge. Doch genügt wiederum ein plötzliches Anrufen oder unerwartete Berührung des Kindes, um die Herzthätigkeit bedeutend zu erhöhen, wobei zugleich Uebelkeit und der Wunsch sich binzulegen eintreten.

Ungeachtet der ailgemeinen Besserung und der vollkommen befriedigenden Ernäbrung - kamen im Laufe des letzten Jahres allmählich noch einige objective Erscheinungen von grösserer Bedeutung zum Vorschein. Während der Fieberparoxysmen nehmlich stellten sich Empfindlichkeit des linken Plexus brachialis und des linken zweiten Intercostalraumes und eine sehr stark ausgesprochene Labilität der Pupillengrösse ein. Die beiden ersten Erscheinungen dauern auch nach den Anfällen fort, sind aber dann bedeutend schwächer ausgeprägt. Wenn wir nun das Auftreten dieser Erscheinungen nur in das letzte Lebensjahr verlegen, so sind wir doch weit entfernt davor, ihr Vorhandensein in den früheren Perioden der Krankheit zu leugnen. Es ist sebr wahrscheinlich, dass sie auch schon fräher existirten, jedoch von uns nicht bemerkt wurden.

Sowohl in diesem Falle, als auch im vorhergehenden, lenkt das plötzliche Erscheinen der Temperaturerhöhung mit gleich- 
zeitiger Beschleunigung der Heizthätigkeit, ohne dass irgend welche wahrnehmbare Ursachen für die Erklärung einer solohen Temperatur vorhanden wären, unsere Aufmerksamkeit auf sich. Im frühesten Alter, wo der Einfluss der psychischen Momente schwerer verfolgt werden konnte, wurde jede Temperaturerhöhung gern durch Erkältung oder durch den anormalen Zustand des Verdauungsapparates erklärt, zumal das Letztere durch das oft gleichzeitige Vorhandensein von Meteorismus gleichsam bestätigt wurde. Allerdings sprach das schnelle Sinken der Temperatur bis zur Norm ohne jegliches Medicament oder nach einer Gabe von Bromkalium gegen die Möglichkeit einer solchen Voraussetzung, aber dieses post hoc konnte dennoch kein volles Recht auf das propter hoc geben. Der im 4. Lebensjahre eingetretene und mehr als 2 Wochen dauernde Anfall liess uns wiederum an die Möglichkeit einer Infectionskrankheit denken; als aber nach 3-4 Tagen diese Voraussetzung weder durch den Charakter der Temperatur; noch durch die objectiven Erscheinungen bestätigt wurde, durften wir an eine Meningitis tuberculosa denken. Diese Annahme konnte für den Augenblick die vorhergegangenen Anfälle erklären und wurde selbst durch das Erbrechen und eine ganze Reihe nervöser Symptome bestätigt. Aber anch dieser Gedanke musste aufgegeben werden, da gar keine Kopfschmerzen vorhanden waron, und das Erbrechen nur beim Versuche sich aufzurichten oder sich zu setzen auftrat; ausserdem sprach auch die Dauer der ganzen Krankheit gegen Meningitis tuberc. Der Einfluss der verticalen Lage auf das Erbrechen und das Aufhören desselben nach Champagner wiesen auf einen anämischen Zustand des Gehirns hin.

Je älter das Kind wurde, desto mehr offenbarte sich die nervöse Anlage desselben, desto deutlicher wurde der Einfluss der psychischen Momente. Bereits im 5. Lebensjahre. konnte der Gedanke, dass die Erscheinungen alle centralen Ursprunges seien, nicht bestritten werden. Jede Aufregung, jeder Kummer zog entschieden eine Temperaturerhöhung nach sich, wobei immer Herzklopfen, zuweilen auch Verdauungsstörungen, Salivation, Schweiss u. s. w. eintraten.

Eine Vergleichung dieses mit dem vorhergehenden Falle zwang sich unwillkürlich auf. Verschieden ihrem Wesen nach, 
hatten sie bloss ein Symptom gemeinsam - die scheinbar ohne Grund eingetretene Temperaturerhöhung. Der zweite Fall war übrigens begreiflicher, obgleich auch hier der Grund und die Ursache der thermischen Erscheinungen nicht minder dunkel blieben. Allerdings wiesen die den Anfall bedingenden Momente, die erwiesene Nervosität des Kindes und andere Erscheinungen auf das Nervensystem, als auf den Ausgangspunkt hin. Der Ausdruck "Nervensystem" konnte jedoch zu jener Zeit nicht näher erklärt werden. Die Literaturangaben waren ebenso allgemein. Wir mussten daher wieder zur - "Individualität" unsere Zafucht nehmen, obgleich wir es dieses Mal zögernd thaten. Und in der That konnten das Krankheitsbild, wie es sich späterhin gestaltete, und die in letzter Zeit angesammelten Beobachtungen als gewisses Kriterium dienen.

Ohne uns auf die näheren Details, von denen weiter unten die Rede sein wird, einzulassen, wollen wir zum dritten Falle, der in das Jahr 1881 fällt, übergehen.

F all IIT. Jul. A., 8 Jahre altes, gut genährtes Nädchen von scheinbar blühender Gesundbeit. Die Mutter anämisch und nervös; der Vater stammt aus einer mit starker Nervosität belasteten Familie und stellt selbst den Typus eines Neuropathen auf anämischer Grundlage dar. Er ist blass, mager und beständig von einer höchst verantwortlichen geistigen Arbeit in Anspruch genommen, leidet sehr oft an Migräne, die ihm nur selten $2-3$ Wochen Ruhe gewährt. Die Lebensbedingungen der Familie waren der Art, dass das Kind sich von frühester Jugend an ausschliesslich unter Erwachsenen befand und Tage lang den mannichfaltigen und nicht immer für Kinder passenden Gesprächen zubörte. Das Resultat einer solchen Erziehung war, dass das Kind trotz seiner 8 Jahre sich mit jedem $13-14$ jährigen Mädchen in Beziehung verschiedener Kenntnisse und Lebenserfahrung messen konnte. Von der Natur mit scharfer Beobachtungsgabe ausgestattet, eignete sie sich sehr schnell die Sprache der Andeutungen an und wuchs zu einem typischen "enfant terrible" heran, in welchem Gutmüthigleit, höchste Eigenliebe and Enthaltsamkeit sich vereinigten. Die sinnliche natürlich unbewusste - Richtung manifestirte sich schon in den, von ihr bereits im 8 . Jahre verfassten, allerdings vollkommen kindlichen Romanen, welche von den Handlungen zweier Helden ausgefültt werden.

Am 10. November 1881 erkrankt das Kind mit fieberhaften Erscheinungen, klagt über grosse Müdigkeit; legt sich jeden Augenblick hin, ist leicht erregbar und leicht zum Weinen zu bringen. Die Miutter legte auf diese Symptome keinen grossen Werth und gab dem Kinde einige Gran Chinin. Dieser Zustand hielt einige Tage an. Die zu verschiedenen Zeiten 
vorgenommenen Temperaturmessungen zeigten grosse Schwankungen zwischen 37 und $39^{\circ}$. Der am 4. Tage consultirte Arzt fand keinerlei Veränderungen und sprach sich für Intermittens aus, da er dazu einige Berechtigung in den starken Temperatursebwankungen zu haben glaubte. Chiningaben von 5 und 10 grm blieben jedoch ohne Erfolg. Gegen den 16. November stellte sich ein trockener, kurzer und fast constanter Husten ein, der die Kranke am Tage belästigte. Sie blieb die ganze Zeit über auf den Füssen und bewahrte ihren Appetit; im Kopf weder Eingenommenheit, noch Schmerz. Dieser Zustand währte bis zum 24. November, an welchem Tage wir die Kranke zum ersten Mal besuchten.

Da ich das Kind rom 3. Lebensjahre an kannte, war ich äusserst verwundert, es in einem höchst erregten Zustande, verbunden mit einer unglaublichen Beweglichkeit, zu finden. Sich selbst überlassen wechselte das Kind jeden Augenblick den Platz, bald sprang es auf, bald legte es sich hin, bald ging es herum fortwälrend das Gespräch wechselnd and antwortete sehr erregt auf die gestellten Fragen.

Die Pupillen sind gleichmässig erweitert, fallen aber bei langer Beobachtung durch ihre Beweglichkeit auf, bald erweitern sie sich ad maximum, bald verengern sie sich ad minimum - ganz unabhängig von der Beleuchtung. Keine Lichtscheu vorhanden. Zuweilen lässt sich eine momentane Bewegung der Augen bemerken, die an Nystagmus erinnert. Dies Alles zusammengenommen verleiht dem Gesicht einen eigenthümlichen, auffallenden Ausdruck. Dabei ist das Gesicht leicht gedunsen, die Wangen stark geröthet; zuweilen aber vollkommen blass. Die Haut fühlt sich eher kalt an.

Der linke Plexus brachialis ist sehr empfindlich auf Druck und der Schmerz strahlt bis in die Schulter und die Hand aus. Der zweite linke Intercostalraum und der anliegende Theil des Sternums sind ebenfalls druckempindindich. Die Kniephänomene und die reflectorische Erregbarkeit der Muskeln sind bedeutend verstärkt.

Die Lungen vollkommen normal. Die Herzdimensionen ebenfalls normal. Die Töne rein und klar. Der Herzstoss verstärkt und frequent. Der Puls $130-140$, fadenförmig, nach 15-20 Schlägen erfolgt eine Intermission. Die Leber und Milz nicht vergrössert, zeigen keine Empfindlichkeit. Der Intestinaltractus in vollkommenster Ordnung; Appetit eher vergrössert; die Zunge rein. Eine genaue Harnanalyse führte zu keinen Resultaten. Die angeführten Symptome schlossen die Möglichkeit irgend einer Infectionskrankheit mit Bestimmtheit aus, liessen jedoch die Frage über die Erhöhung der Temperatur, die sich den Aussagen der Angehörigen gemäss, bereits 14 Tage auf mehr oder weniger hohen Ziffern hielt, unbeantwortet. Da auch die geringste Milzanschwellung fehlte und ebenso jeder Anhaltspunkt für eine Malariainfection, so konnten wir auch Intermittens ausschliessen. Die vom 24. ab vorgenommene regelmässige tägliche Temperaturmessung gab nachstehende Curve. 
Fig. 4.

November 1881.

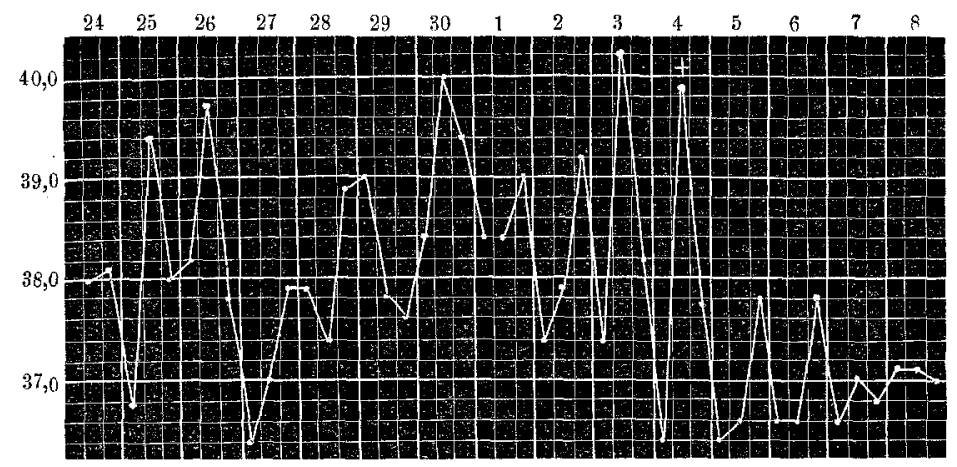

Der oben erwähnte Husten dauerte vom 24. November bis zum 4. December. Die Herzthätigkeit erhielt sich auf derselben Höhe, 130-140 Herzschläge in der Minute. Das Kind war die ganze Zeit über auf den Füssen und brachte keine Klagen vor. Die Antipyretica blieben ohne jeglichen Erfolg: so wurden z. B. am 28. November, um 10 Uhr Morg. 10 gr Chinin und um 4 Uhr $5 \mathrm{gr}$ gereicht und dennoch stieg die Temperatur um $8 \mathrm{Uhr}$ Abds. bis 38,90. Am 29. Morg. war die Temperatur 39, $1^{0}$; das Chinin wurde durch anhaltende ( $\frac{1}{2}$ Stunde) Bäder von $27^{\circ}-26^{\circ} \mathrm{R}$. ersetzt; solcher Bäder wurden 3 gemacht: um 3 Uhr Nachm., 10 Uhr Abds. und um 2 Uhr Nachts; nichtsdestoweniger war die Temperatur am Morgen des 30 . bis $40^{\circ}$ gestiegen. An diesem Tage wurden 2 Bäder gemacht und 2 Gaben Natr. salicyl. zu $10 \mathrm{~g}$ gereicht - und demnach zeigte das Thermometer um $4 \mathrm{Uhr}$ 40,2 $2^{\circ}$. Am 4. wurde Inf. digital. abwechselnd mit Bromkalium verordnet; gleichzeitig begann die Temperatursenkung und das Versehwinden der angegebenen Symptome, so dass am 10. December der beständige Wechsel der Pupillengrösse, und zum 15. die Empfindlichkeit des linken Plexus brachialis und des zweiten linken Intercostalraumes verschwanden.

Im Juli 1882 klagte das Mădchen, welches am Morgen vollkommen gesund war, am Nachmittag über Unwohlsein, wobei sich zugleich die schon bekannte Reizbarkeit und Unstätigkeit äusserten. Ganz zufällig sahen wir das Kind um diese Zeit und konnten eine stark ausgeprägte Labilität der Pupillen und einen frequenten kleinen Puls, 120 Schläge, constatiren, die Empfindlichkeit im zweiten linken Intercostalraum war nicht vorhanden. Die sogleich vorgenommene Temperaturmessung ergab 39,2 $2^{\circ}$ Unter der Anwendung von Bromkalium sank die Temperatur gegen Abend bis $38^{\circ}$ und am folgenden Morgen war sie blos $36,8^{\circ}$.

Im December 1882 wurde das Kind wieder krank und ungeachtet der sofort aufgetretenen üblichen Erseheinungen, vielleicht auch Dank eben dieser "gewöhnlichen" Erscheinungen, wurden wir erst am 6. Krankheitstage benachrichtigt. Die Temperatur stieg auch dieses Mal bis 40,30 maximum. 
$A$ us gewissen, weiter unten zu erwähnenden Gründen verordneten wir Sauerstoff, wonach die Temperatur raseh sank und überhaupt sehr bald Besserung eintrat.

Im Februar 1883 stellte sich von Neuem ein Fieber von 3tägiger Dauer ein. Dieser Paroxysmus war durch Unannehmlichkeiten in der Schule und gekränkte kindliche Eigenliebe hervorgerufen. Am zweiten Tage stieg die Temperatur bis $39,3^{\circ}$. Unter dem Einflusse von Sauerstoff sank sie gegen Abend bis $37,9^{\circ}$ und gegen Mittagszeit, am dritten Tage bis $36,8^{\circ}$.

Wenn wir nun diesen Fall mit dem vorhergehenden, zweiten, vergleichen, finden wir schon mehr Gemeinsames, als zwischen dem zweiten und ersten Falle. In beiden zeigen sich dieselben 2 Cardinalerscheinungen - eine erhöhte Temperatur und Veränderungen der Herzthätigkeit, bei gleichen objectiven Erscheinungen von Seiten des Nervensystems. In beiden Fällen wird Labilität der Pupillen, Empfindlichkeit des 2. linken Intercostalraumes und des linken Plexus brachialis constatirt, wobei keine erklärenden Ursachen zu finden waren.

Ungeachtet dieser Identität der hervorragenden Erscheinungen liess uns der erste Paroxysmus vom 10. November 1881 dennoch an die Möglichkeit einer beginnenden Meningitis tuberculosa denken, wofür theilweise 2 Fälle von Phthisis in der Familie des Vaters sprachen, oder an einen Prozess in den Bronchialdrüsen, worauf vielleicht der die Kranke belästigende, trockene, kurze Husten hinwies. Unter diesen diagnostischen Schwankungen und fortwährenden nutzlosen Versuchen, die Temperatur zum Sinken zu bringen, verstrich die Zeit bis zum 4 . December, wo zu rein palliativen Zwecken Digitalis und Bromkalium gereicht wurden. Gleich darauf fällt die Temperatur rasch bis zur Norm und es verschwinden alle Erscheinungen. Die folgenden Anfälle, welche stets dasselbe Bild darboten, wiesen bereits direct auf den Sitz der Krankheit hin. Der offenbare Einfluss psychischer Affecte, als Krankheitserzeuger in der Mehrzahl der Anfälle, die objectiven nervösen Erscheinungen, der Husten, welcher einerseits in scheinbarem Zusammenhang mit der Temperatursteigerung, anderseits mit dem Herzklopfen stand, der Zustand der Pupillen - dies alles gab bereits ein Recht sämmtliche Erscheinungen zur Kategorie der "centralen" zu rechnen. Der Umstand, dass die keinem antipyretischen Mittel weichende Temperaturerhöhung nach Bromkalium und Sauerstoff fiel, bestätigte noch diese Annahme. 
In Anbetracht der 2 vorhergehenden Fälle war die Frage von einer "Zufälligkeit" oder „Individualität" wohl kaum zulässig. Die Identität der Cardinalerscheinungen schien die Annahme eines, für alle 3 Fälle gemeinsamen Ausgangspunktes der thermischen Erscheinungen zu berechtigen. Es war klar, dass die Ursache in centralen Sphären zu suchen ist und dass diese Ursache, nach den Folgen zu urtheilen, offenbar eine functionelle war.

Den vierten Fall haben wir im Jahre 1883 beobachtet.

Fali IV. Die Kranke, L. W., wandte sich zum ersten Yale an mich am 12. März 1883 mit Klagen uber heftige Kopfschmerzen, welche von Zeit zu Zeit auftreten und jedes Mal circa 1 Monat anhalten. Diese Kopfschmerzen sind ungewöhnlich stark, verursachen oft vorübergehende Bewusstlosigkeit und Delirien und sind dadurch ausgezeichnet, dass im Verlauf eines und desselben Tages die heftigsten Anfälle mit freien Intervallen, von zuweilen stundenlanger Daner, wechseln. Die Krankheit besteht bereits lange, seit ungefähr 8-10 Jahren; die Kranke hat sich an die Anfälle von mittlerer Stärke bereits so gewöhnt, dass dieselben nicht störend auf ihre Lebensweise einwirken.

Patientin ist 24 Jahre alt, unverheirathet; von sehr hohem Wuchse (2 Arsch. $8 \frac{1}{2}$ Wersch. $=180 \mathrm{~cm}$ ) von gut entwickeltem Knochenbau, aber mit welker Musculatur; das subcutane Fettpolster mässig entwickelt, keine Zeichen von Abmagerung vorhanden. Das Gesicht ist ziemlich voll, aber sehr blass und spielt leicht in's Wachsfarbene, die Schleimhäute ebenfalls blass. Wenn Pat, aufgeregt ist oder wenn sie spricht, so kommt ein rascher Wechsel von Röthe und Blässe des Gesichtes zum Vorschẹin.

Die Brust ist gut entwickelt. Respiration besehleunigt, 24, oberflächlich. Lungen normal.

Die Herzdimensionen ebenfalls normal. Der Herzstoss ist verstärkt und beschleunigt, mit metallischem Beiklang. Ein minutenlanger mässig schneller Gang im Zimmer genügt, um die Frequenz des Pulses um 20 Schläge in der Minute und anch die Respiration bedeutend zu beschleunigen. Die Herztöne rein, der zweite Pulmonalton etwas verstärkt. Geräusche sind weder am Herzen, noch an den Gefässen wahrzunehmen.

Die Unterleibsorgane gesund. Der Darmtractus functionirt regelmässig, wenn man von einer unbedeutenden Neigung zu. Verstopfungen absieht. Der Urin ist normal. Von Seiten der Geschlechtstheile sind auch keine Abweichungen vorhanden.

Pat. ist von frühester Kindheit an sehr empfänglich für äussere Eindrücke und leicht reizbar gewesen; ihr unstetes und ungestümes Wesen haben sie stets in der zahlreichen Familie ausgezeichnet; der geringste Kummer, die kleinste Freude machten sie krank, indem bald Kopfschmerz, bald Herzklopfen eintraten. Nach einer Reihe schlafloser Nächte und sehr ermüdender Beschäftigung (Examen) stellten sich bei dem 16jährigen Mäc- 
chen heftige Kopfschmerzen ein, welche keiner Behandlung weichen wollten und schon sehr bald von einer Gesichtsverzerrung begleitet wurden. Die damals behandelnden Aerzte befürchteten ernste Folgen. Ein Aufenthait im Auslande jedoch beseitigte die Erscheinungen in verhältnissmässig kurzer Zeit obne jegliche Behandlung und von Gesichtsverzerrung ist jetzt keine Spur mehr vorhanden. Das Gedächtniss ist in den letzten Jahren geschwächt, das Gelesene wird mühevoller aufgefasst.

Neben diesen subjectiven Erscheinungen seitens des Nervensystems sind auch folgende objective Erscheinungen wahrnehmbar. Die Pupillenweite ändert sich jeden Augenblick. Der linke Plexus brachialis ist stark empfindlich; eine gleiche Empfindlichkeit existirt auch in zweiten linken Intercostalraum. Das Kniephänomen stark ausgeprägt. Die reflectorische Contractilität der Huskeln ist erhöht; leichte Schläge mit dem Percussionshammer rufen eine starke Contraction hervor, am ausgesprochensten an den Muskeln der oberen Extremitäten. Die Extremitäten sind kalt. Hautsensibilität normal.

Die Kranke stammt aus einer reichen, intelligenten Familie, welche in den letzten 4-5 Jahren von sehr vielen moralischen Unglücksfällen heimgesucht worden ist. Der Tod beider Eltern im Jahre 1881 erschütterte die Kranke in hohem Grade. Der Vater litt an Asthma und starb an einem Herzfehler; die Hutter war das ganze Leben hindurch nervös. Eine der Schwestern, die jüngste, zeigt alle Erscheinungen einer beginnenden Graves'schen Krankheit; eine andere beständig im Auslande lebende Schwester leidet augenblicklich an derselben Krankheit. Von den 3 Brüdern kann nur einer gesund genannt werden.

Dieses war ungefähr der Status der Kranken bei unserer ersten Untersuchung; unsere Aufgabe bestand für's Erste nur darin, der Pat. eine Erleichterung gegen heftige Kopfschmerzen, welche eben begonnen hatten, zu verschaffen. Abgesehen von der allgemeinen Behandlung, die gegen Anämie gerichtet war, verordneten wir allgemeine warme Bäder (280); schon bald (nach der dritten Wanne) liess der Anfall nach.

10 Tage danach, nebmlich am 22. März wurde zum ersten Male eine Temperatursteigerung constatirt - $38,4^{\circ}$ am Abend; zugleich stellt sich ein heftiger, trockener Husten ein, der sich bei der geringsten Bewegung verstärkt. In der Nacht ist der Husten geringer, am Tage stärker; am Morgen, beim Aufstehen, ist er am stärksten. Eine genane Untersucbung der Lungen giebt absolut negative Resultate. Das Herz aber befindet sich in einem äusserst gereizten Zustande; die geringste Bewegung genügt, eine bedeutende Athemnoth und Beschleunigung der Herzthätigkeit hervorzurufen. Die Töne sind rein und stark. Die Frequenz des Pulses steigt leicht bis 120. Die gebräuchlichen Mittel gegen den Husten hatten keinen Erfolg. Am 25. erreichte der Husten eine bedeutende Stärke und wurde sogar durch kurzes Gespräch hervorgerufen. Der Lungenbefund bleibt derselbe. Das Herz zeigt dieselbe Gereiztheit; die Empfindlichkeit im linken zweiten IntercostaIraum und im angrenzenden Theile des Sternums aber ist jetzt bedeutend stärker; auch der linke Plexus brachialis ist jetzt empfindlicher gegen Druck; letzterer 
mit dem Finger ausgeführt, ruft einen heftigen Schmerz im ganzen Arm bis zu den Fingerspitzen hervor.

Zu gleicher Zeit wurde bemerkt, dass die verticale Lage einen Hustenanfall bedingt, wodurch sich vielleicht die Abnahme der Hustenstärke während der Nacht erklären lässt.

Diese Thatsachen dienten uns als Ausgangspunkt für die Behandlungsweise. Horizontale Lage, Bromkalium und Chinin. bromat., Convallar. majal. führten eine bedeutende Erleichterung berbei.

So ging es bis zum 1. April, als abermals gegen Abend eine Temperatursteigerung constatirt wurde (die mittlere Norm für unsere Kranke war $37^{0}$ Morg., 37,40 $-37,5^{0}$ Abds.). Ein Versuch, durch Chin. mur. die Temperatur zum Sinken zu bringen, blieb ohne Erfolg; am schnellsten, wenn auch ebenfalls langsam, wirkte in dieser Hinsicht Chin. brom. Vom 4. April wurde eine regelmässige Temperaturmessung angestellt, welche folgende Curve gab.

Fig. 5.

April 1883.

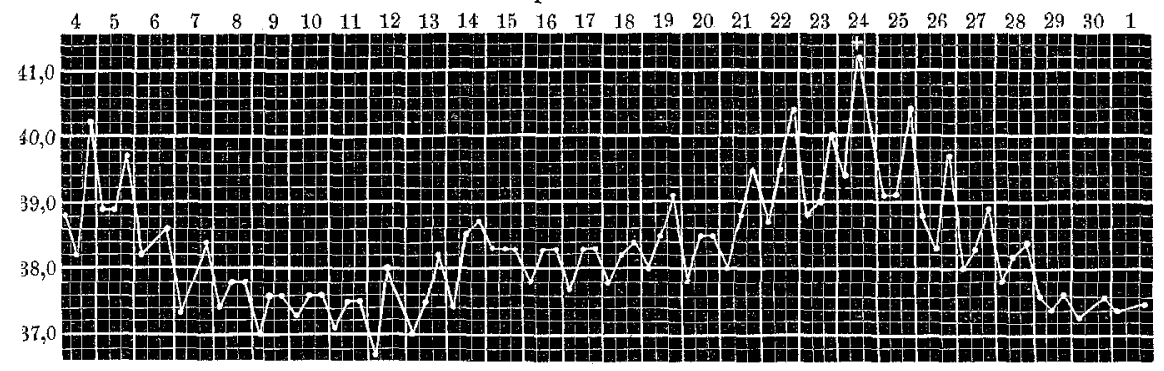

Am Morgen des 4. stieg die Temperatur bis $38,8^{\circ}$. Der Lungenbefund bleibt normal; die Herzthätigkeit sehr beschleunigt; der Querdurchmesser des Herzens ist kaum vergrössert, an der Basis ein deutliches systolisches Geräusch, in den Halsvenen wird jetzt Nonnengeräusch wahrgenommen; Puls 120, klein, fadenförmig, leicht comprimirbar. Ausserdem klagt Pat. über Schmerz und eine Schwellung im rechten Oberschenkel; das Anschwellen begann angeblich erst am vorhergebenden Abend, hat jetzt bereits bedeutende Dimensionen erreicht, so dass am Abend der Unfang des Oberschenkels im oberen Drittel um $8 \mathrm{~cm}$ grösser ist, als am gesunden Beine. Wie es sich jetzt erst erweist, erkrankt das rechte Bein bereits zum 3. Male in den letzten 5 Jahren. Zum ersten Male schwoll der rechte Oberschenkel an, im Jahre 1877, im Anschluss an eine acute, 14 Tage dauernde Lungenentzündung, und die Diagnose wurde damals auf Venenthrombose gestellt. Die Folge dieser Thrombose waren Ulcera am rechten Oberschenkel (im Jahre 1878), die Spuren dieser Ulcera sind noch jetzt als breite Narben sichtbar. Endlich, im Jahre 1881, führte langes Stehen (Pflege der kranken Mutter) eine Thrombose des rechten Unterschenkels herbei, welche von Prof. Billroth constatirt wurde und deren Folgen sehr lange bemerkbar waren.

Archiv f. pathol. Anat. Bd. XCVI. Hft. 1. 
Am Abend desselben Tages war die Temperatur 40,20.

Am Morgen des 5. April 38,90. Das Bein ist in statu quo; der Schmerz nicht bedeutend. Husten, Herzklopfen und Asthma auch in statu quo. Der consultirte Chirurg constatirte blos eine bedeutende Bärte des angesehwollenen Oberschenkels und stellte die Diagnose sub dubio, da er gar keine Zeichen einer Phlebitis oder Lymphangoitis constatiren konnte. Es wurden Rube und kalte Umschläge verordnet, innerlich bekam die Kranke wie früher Chin. bromatum nnd Convallar. majal. nur in grösseren Dosen. Darauf beginnt die Temperatur rasch zu fallen (s. die Curve), zu gleicher Zeit nimmt auch die Geschwulst des Oberschenkels rasch ab, so dass gegen den 9. die Temperatur normal wurde und die Folgen der Thrombose geschwunden waren.

Vom 9. bis zum 13. April blieb die Temperatur normal, der Husten und Herzklopfen waren um Vieles geringer.

Am Abend des 12. stieg die Temperatur von Nenem bis $38^{\circ}$. Die Herzthätigkeit erhöht. Die Geräusche sehr stark ausgeprägt. Der Husten stärker.

Am 13. April Menstrua, zur regelmässigen Zeit und schmerzlos.

Vom 13. bis zum 21. bleibt die Temperatur um circa $1^{0}$ erböht. Die Menstrua sind während dieser ganzen Zcit äusserst gering, bleiben Tage lang g̈̈nzlich aus. Der Husten verstärkt sich.

Am 21. wird zum ersten Mal ein kleines, schwaches, kaum wahrnehmbares Geräusch an der Herzspitze bemerkt; es hat eher einen präsystolischen Charakter. Der Querdurchmesser des Herzens ist un ein Geringes vergrössert. Die recbte Grenze dieses Durchmessers ist übrigens sehr veränderlich, indem sie im Laufe eines und desselben Tages recht bedeutende Schwankungen zeigt. Puls 120. Keine Arhythmie vorhanden. Die Geräusche an der Basis und in den Halsvenen sind sehr stark. Um 4 Uhr Nachm. stellt sich Schittelfrost bei $38,8^{\circ}$ ein; am Abend $39,4^{\circ}$. Ein gegeringer Schmerz im linken Bein.

22. April Morg. $38,7^{\circ}$. Mitten am Tage Scbüttelfrost bei $39,6^{\circ}$; am Abd. 40,40. Grosse Gaben Chinin (bis $20 \mathrm{~g}$ ) und Natr. salicyl. ( $4 \mathrm{Mal}$ zu $10 \mathrm{~g}$ ) hatten auch nicht den geringsten Effect. Unstillbarer Husten lässt sogar bei horizontaler Lage nicht nach. Der Lungenbefund normal. Puls 120. Inf. convallar. majalis.

Am Morgen des 23. wird ausserdem Inf. digital. (e. gr. xij) verordnet. Die Temperatur am Tage 39,9 $9^{\circ}$, am Abd. 39,40. Die Leber und Milz sind absolut normal. Die Zunge etwas belegt. Stuhl normal. Puls 112.

24. April Morg. $39,4^{0}$. Mitten am Tage Schüttelfrost, Erbrechen

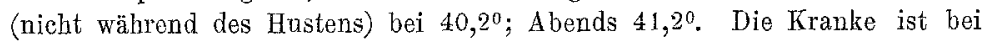
völliger Besinnung, spricht mit der Umgebung. Die Nächte sind vom 21. an schlaflos. Der Husten und Herzklopfen sind äusserst quälend. Der linke Oberschenkel und zum Theil auch der Unterschenkel sind heute geschwollen. Der Umfang des linlen Unterschenkels in der Mitte $34 \mathrm{~cm}$; rechts 32; der linke Oberschenkel in der Mitte $47 \mathrm{~cm}$, rechts $46 \mathrm{~cm}$. Aus 
weiter unten anzuführenden Gründen wurde hente ein Gemenge von Sauerstoff mit Stickstoffoxydul zum Einathmen verordnet. Von 4 Uhr Nachm. bis zum Morgen des 25. hat die Kranke 8 Cubikfuss von diesem Gemenge verbraucht.

25. Mai Morg. $40^{\circ}$; Mittags $39^{\circ}$, Abds. 40,40. Der Husten um Vieles geringer. In der Nacht kurzer Schlaf. Der linke Untersehenkel $35 \mathrm{~cm}$, der Oberschenkel 48. Das Einathmen von Sauerstoff wird fortgesetzt. Puls 108.

26. April. Die Kranke hat 8 Cubikfuss Sauerstoff eingeathmet. Morg. $38,8^{\circ}$, Mittags $38,2^{0}$, Abds. 39,6 . Die Nacht hat sie besser verbracht. Der linke Unterschenkel $36 \mathrm{~cm}$; der Oberschenkel $49 \mathrm{~cm}$. Der Husten und Her\%klopfen bedeutend geringer. Puls 96. Das Einathmen von Sauerstoff, zu 8 Cubilfus, wird fortgesetzt.

27. April Morg. 380, Abds. 38,80. Puls 96. Der Husten ist fast verschwunden. Der liake Unterschenkel $35 \mathrm{~cm}$, der Oberschenkel $48 \frac{1}{2} \mathrm{~cm}$.

28. April. Die Temperatur sinkt beständig. Das Anschwellen des Beines nimmt $\mathrm{zu}$. Unterschenkel $35 \mathrm{~cm}$, Oberschenkel $49 \frac{1}{2}$; im oberen Drittel ist der linke Oberschenkel heute besonders geschwollen, der Unterschied im Vergleich mit dem rechten Oberschenkel beträgt $8 \mathrm{~cm}$ (r. $48 \mathrm{~cm}, 1.56)$.

29. April. Kein Husten mehr. Der Schlaf bedeutend besser. Sauerstoff wird weiter gebraucht. Die Geschwulst des Beines ist geringer; der linke Oberschenkel $47 \mathrm{~cm}$, im oberen Drittel $54 \frac{1}{2} \mathrm{~cm}$.

2. Mai. Normale Temperatur. Die Geschwulst nimmt allmählich ab.

4. Mai. Puls 80. Das Geräusch an der Herzspitze ist kaum hörbar, an der Basis aber noch ziemlich stark. Pat. hat das Bett verlassen. Es wurde Eisen und Arsen verordnet.

1. Juli. Die Kranke hat sich erholt und hat zugenommen. Die anämischen Geräusche sind verschwunden.

Der Beginn der Krankheit in diesem Falle bietet gar keine Schwierigkeiten. Es war klar, dass wir es mit einer stark ausgesprochenen Chlorose zu thun hatten.

Vom 22. März bis 1. April war der Husten das einzige hervorragende Symptom. Derselbe war für die Kranke sehr lästig und stand in völligem Widerspruch mit dem absolut normalen Lungenbefund. Sein sichtbarer inniger Zusammenhang mit dem Herzklopfen und seine Zunahme bei verticaler Lage wiesen, wenn auch nur annähernd, auf den causalen Ursprung hin und widersprachen der gestellten Diagnose nicht.

Die am 1. April eingetretene, wenn auch relativ unbedeutende Temperatursteigerung (die hohe mittlere Norm) brachte schon diagnostische Schwierigkeiten mit sich, besonders als am 4. die Temperatur bis $40^{\circ}$ stieg. Der weitere Verlauf führte noch zu weiteren Zweifeln, — es wurde an die Möglichkeit einer 
Endocarditis ulcerosa, oder auch einer perniciösen Anämie gedacht.

Weiter unten, bei der genaueren Besprechung dieses Falles, werden wir uns bemühen, unsere Gründe für die Differentialdiagnose auseinanderzusetzen, hier sei es nur bemerkt, dass wir unabhängig von diesen 2 Wahrscheinlichkeitsdiagnosen noch an eine Möglichkeit gedacht haben, indem wir die Symptome der früher beschriebenen 3 Fälle, mit denen dieser Fall vieles Gemeinsames hatte, im Auge behielten. Uns auf manche Einzelheiten basirend, hielten wir es für möglich, das ganze Krankheitsbild aus centralen Ursachen zu erklären. Von diesen, vielleicht verfrühten Combinationen ausgehend, beschlossen wir, wie es auch in jenen Fällen geschah, Sauerstoff anzuwenden. Das Resultat ist aus der Curve ersichtbar.

So gering das von uns angeführte Material auch ist, so erhalten wir doch aus diesen 4 Fällen eine ganze Reihe von identischen Erscheinungen und damit zugleich ein Recht, letztere näher zu analysiren und zu untersuchen, ob diese, dem Anschein nach, ähnlichen Erscheinungen auch einen gemeinsamen Ausgangspunkt haben. Aus diesem Grunde erlauben wir uns alle unsere Fälle in derselben Reihenfolge einzeln einer Analyse zu unterwerfen.

Fall I. Es wurde bereits erwähnt, dass wir in der Literatur gar keine Angaben für die Möglichkeit einer so hohen Temperatur während einer diphtheritischen Paralyse fanden und daher gezwungen waren, uns mit der damals scheinbar einzig möglichen Erklärung zu begnügen: Zufall oder Individualität.

Wenn wir nun jetat diesen Fall auf Grund des angesammelten Materiales betrachten, so müssen wir uns natürlich die Frage vorlegen, ob nicht das Wesen der diphtheritischen Paralyse selbst in dieser Zufälligkeit eine gewisse Rolle spiele?

Aus der hierher gehörigen Literatur ersehen wir, dass die meisten Autoren von der Temperatur bei den Lähmungen diphtheritischen Ursprunges gar nichts erwähnen. Nur Sanné ${ }^{i}$ ) giebt an, dass "sehr oft der Anfang der Lähmung durch Fie-

1) De la diphthérie. 1877. 
berzustand manifestirt wird"; dieselbe Ansicht theilen auch Lorain und Lepine ${ }^{1}$ ), indem sie mittheilen, dass der Anfang der Lähmung zuweilen durch Fieber sich kundgiebt, fügen aber gleich hinzu, dass das Fieber dabei durchaus nicht beständig sei. Maingault ${ }^{2}$, dessen Competenz in dieser Frage wohl kaum bezweifelt werden kann, leugnet, im Gegentheil, das Vorkommen des Fiebers. Wenn auf die Weise die Literaturangaben Fieberbewegungen bei diphtheritischen Lähmungen nicht unbedingt in Abrede stellen, so erscheinen dieselben jedenfalls mehr als zweifelhaft, da selbst nach Sanné, Lorain und Lepine das Fieber blos den Anfang der Lähmungen bezeichnet. Von dieser Seite her konnten wir also keine Erklärung für unseren Fall finden.

Ferner blieben wir natürlich bei dem Gedanken stehen, ob sich nicht im pathologisch-anatomischen Befunde eine, wenn auch indirecte Erklärung finden liesse. Aber auch in dieser Beziehung giebt die Literatur wenig Definitives.

Biș zum Jahre 1862 betrachtete man die diphtherischen Lähmungen als Typus der Lähmungen „sine materie“. Die in diesem Jahre zuerst von Charcot und Vulpian ${ }^{3}$ ) in einem Falle constatirte Atrophie der Muskelnerven des Gaumensegels diente als Ausgangspunkt einer ganzen Reihe von Untersuchungen, welche den Zweck hatten, das Vorhandensein materieller Hirnveränderungen als Grund der in Rede stehenden Lähmungen zu beweisen. Buhl ${ }^{4}$ ) beschrieb im Jahre 1867 Blutextravasate mit Gehirnerweichung und Veränderungen in den vorderen und hinteren Wurzeln des Rückenmarkes; die Stränge waren verdickt in Folge des Vorhandenseins von Kernen im Bindegewebe, welche denen analog waren, die Buhl in diphtheritischen Membranen gefunden hatte, und die er als charakteristische für dieșen Prozess bezeichnete. Im Jahre 1871 fand 0 ertel $^{5}$ ) Blutextravasate

1) Die Aufsätze „Diphthérie" in Nouveau dictionnaire de médecine. Bd.XI. (Ich citire nach Landouzy, Des paral. dans les maladies ajgues. Paris 1880.)

2) De la paralysiée diphthérique. Paris 1860 .

3) Comptes rendus de la Société de Biologie. 1862.

${ }^{4}$ Z Zeitschr. f. Biologie. 1863. (Einiges über Diphtherie).

5) Deutsches Arch. f. klin. Med. Bd.XIV. 1871, S. 242 u. f. 
in dem Subarachnoidalraum, Imbibition der vorderen Hörner und croupöses Exsudat im Rückenmarkskanal. Um dieselbe Zeit constatirte Leyden ${ }^{1}$ ) Veränderungen, welche er unter dem Namen Neuritis migrans beschrieb, und erklärte die Lähmungen dadurch, dass die Veränderungen im Nerven in aufsteigender Richtung die Centren erreichen, und auf diese Weise die Lähmungen bedingen können. Senator ${ }^{2}$ ) nimmt den specifischen Charakter für diese Lähmungen nicht an und schliesst sich der Leydenschen Ansicht an. Vulpian ${ }^{3}$ ) fand bei der Section von 3 an Diphtheritis gestorbenen Kindern gar keine Veränderungen in den Gehirnhäuten; das Einzige, was er bei 2 dieser Sectionen fand, war eine Auflockerung des Bindegewebes im äusseren and hinteren Theile der Vorderhörner und eine Trübung der Nervenzellen an diesem Orte.

Bei Landouzy $y^{4}$ ) begegnen wir einer genauen Wiedergabe der im Jahre 1878 erschienenen Untersuchungen von Déjérine ${ }^{5}$ ). Letzterer weist auf Grund seiner 5 Sectionen auf folgende Veränderungen hin: degenerative Atrophie der vorderen Wurzeln und der intermusculären Nerven, Intactsein der hinteren Wurzeln, unbedeutende Veränderungen der grauen Gehirnmasse bei Unversehrtheit der weissen Substanz. Landouzy selbst schliesst sich der Meinung Déjérine's an und lässt für die Mehrzahl der Fälle eine Neuritis der vorderen Wurzeln mit unbedeutenden Veränderungen der grauen Gehirnmasse zu; die Veränderungen in den Gehirnhäuten aber hält er für zufällige Erscheinungen.

Dies ist, in allgemeinen Zügen, die Summe der pathologischanatomischen Veränderungen, welche man wohl laum als genügend für die Lösung der uns interessirenden Frage bezeichnen kann. Wir beabsichtigen nicht, hier näher auf die diphtheritischen Lähmungen einzugehen, da es uns nur indirect interessirt.

1) Ueber Reflexlähmungen (Volkmann's Samml. klin. Vorträge No. 2). Diesen Gedanken hatte Leyden bereits früher ausgesprochen (Centralbl. f. d. med. Wissensch. 1865).

2) Senator, Dieses Archiv 1872. S. 79.

3) Maladies du système nerveux. 1876.

4) Des paralysies dans les maladies aigues. Paris 1880. p. 285.

5) Recherches sur les lésions du système nerveux dans la paralysio diphthérique. Arch. de physiol. normale et pathol. 1878. 
Wir führten die Literaturangaben nur deswegen an, damit nicht unsere Worte - dass auch in pathologisch-anatomischer Hinsicht die Literatur der diphtheritischen Lähmungen noch sehr wenig bietet - unbegründet erscheinen. Wie verlockend die Leyden'sche Theorie von der aufsteigenden Neuritis auch sein mag, so giebt sie uns doch nur jn den gewöhnlichen Fällen Aufschluss, d. h. in denjenigen, wo Rachendiphtherie von einer Lähmung des Gaumensegels begleitet wird; sie ist jedoch nicht im Stande, diejenigen Fälle zu erklären, wo Lähmungen des Gaumensegels eintreten nach Diphtheritis solcher Theile, welche mit dem Rachen nichts gemein haben. Es genuigt in dieser Beziehung auf die in der Literatur bekannten Fälle von Rachenlähmungen, welche nach Diphtheritis der Haut eintraten, hinzuweisen ${ }^{\mathrm{I}}$ ).

Auf diese Weise bleibt die Frage nach dem Wesen der diphtheritischen Lähmungen unbeantwortet; am wenigsten streitig erseheint noch die Auffassung von $J_{a c c o u d}{ }^{2}$ ), „dass bei dieser Sachlage zugegeben werden kann, dass die Affection des Rachens centripetal auf die Vasomotoren der Nẹrvencentren wirkt, so dass die Ernährung dieser Centren temporär modificirt und dadurch ihre Functionsstärke geschwächt wird". Diese allgemeine These von $\mathrm{Jaccoud}$ findet ihre theilweise Bestätigung in einer Menge klinischer Thatsachen, von denen folgende in den Vordergrund gestellt werden müssen: das Alterniren der Lähmungen, ihr verhältnissmässig rasches Verschwinden und der Charakter selbst, der sich um Vieles von Heerderkrankungen unterscheidet. Nimmt man ferner in Betracht, dass die Lähmungen hauptsächlich den Rachen, die Kehle, Speiseröhre und Trachea betreffen, d. h. die hauptsächlich vom Vagus innervirten Gebiete, so könnte man die Auffassung von Jaccoud noch weiter entwickeln, wenn man zulässt, dass als Ausgangspunkt der diphtheritischen Läbmungen Functionsstörungen im verlängerten Mark und besonders in den Vasomotorencentren und den Vaguscentren dienen. Welche Rolle jedem von ihnen zukommt, lässt sich freilich a priori schwer bestimmen, in Bezug auf den $\mathrm{N}$. vagus jedoch finden wir eine wichtige Bestätigung in der Ar-

1) Paterson, Medical Times. 1866. p. 608.

2) Jaccoud. Bd. III. S. 75. 
beit Revilliod's ${ }^{1}$ ). Indem er die Ursachen, Formen und Folgen der Lungenlähmung bei Diphtheritischen bespricht, gelangt er nach einer Reihe von Beweisen zu dem Schlusse, dass die Lungenentzündung bei diesen Kranken nicht als selbständiger Prozess und nicht als Folge, sondern als Ausdruck des paralytischen Prozesses angesehen werden muss, welcher sich hauptsächlich im Gebiet des N. vagus einnistet. Weiter sagt er: „Der in der Mehrzahl der Fälle durch Pneumonie bedingte Tod lässt sich weniger durch entzündliche Erscheinungen, als vielmehr durch die Lähmung des $\mathrm{N}$. vagus erklären“.

Wenn wir diesen Standpunkt festhalten und die Theilnahme der 2 genannten Centren als bedingende Momente für diphtheritische Lähmungen zulassen, sind wir im Stande, - allerdings können wir uns den Vorwurf der Voreiligkeit zuziehen, - die hohe Temperatur in unserem ersten Falle durch Störung der normalen Thätigkeit des vasomotorischen Centrums zu erklären. Die Möglichkeit dieser Erklärung ist schon durch die Rolle des Wärmeregulators, welche den Vasomotoren zugeschrieben wird, gegeben.

Wenn wir also die Einwirkung des vasomotorischen Centrums zulassen, können wir schon verhältnissmässig leicht die Wirkung des Sauerstoffs in unserem Falle erklären. Es ist bekannt, dass die Erregbarkeit des vasomotorischen Centrums in innigem $\mathrm{Zu}-$ sammenhang mit der Quantität der Gase in dem durch das verlängerte Mark strömenden Blute steht; ferner ist es bekannt, dass dieses Centrum bei Apnoe am wenigstens erregbar ist (Landois); dagegen wird es bei der venösen Beschaffenheit des Blutes stärker gereizt, wodurch eine Contraction aller Arterien hervorgerufen wird (Thiry, nach Landois); ähnlich wirkt auch plötzliche Blutarmuth des verlängerten Markes (Navalichin) und plötzliche Stauung bei venöser Hyperämie. Von diesen physiologischen Combinationen ausgehend, würde es sich annehmen lassen, dass in unserem Falle unter dem Einfluss von Diphtherie Anämie und besonders Anämie des verlängerten Markes sich entwickelt habe. Dadurch wurde das vasomotorische Centrum stark erregt, die peripherischen Gefässe folglich verengt,

1) Diphthérie, croup et trachéotomie. Comptes rendus et mémoires du Congrès international des sciences méd. Genève 1878. p. 181 . 
woraus Temperatursteigerung resultirte. Durch das Einathmen von Sauerstoff bewirkten wir einigermaassen Apnoe, bei welcher die Erregbarkeit des Centrums verringert wird, folglich musste anch die Temperatur sinken.

Fall II und III. Da die meisten Symptome im Falle II und III identisch sind, so werden wir sie gemeinsam besprechen. Uns treten hier, unabhängig von der uns hauptsächlich fesselnden Erscheinung der T'emperaturerhöhung, folgende gleichartige Symptome entgegen: 1) Schmerzhaftigkeit des 2. linken Intercostalraumes; 2) Schmerzhaftigkeit des linken Armnervengeflechtes; 3) Labilität der Pupillen; 4) gesteigerte Muskel- und Sehnenreflexe; 5) Herzklopfen.

Wenn wir nun auch schon früher vermuthen konnten, dass die Ursache des ganzen Prozesses in dem Centralnervensystem liege, - eine Vermuthung übrigens, die mehr durch Ausschluss anderer Ursachen, als durch positive Thatsachen gewonnen wurde, - so betreten wir mit den oben aneinander gereihten Symptomen einen mehr reellen Boden. Es bleibt uns noch, nachdem wir die Frage nach dem Ursprunge entschieden haben, übrig, eine Lösung für die Art und Weise zu suchen, und wir beginnen dabei mit der Analyse der Symptome.

1) Die Schmerzhaftigkeit des 2 . linken Intercostalraumes bildet nach Peter ${ }^{1}$ ) oines der hervorstechendsten Symptome bei Angina pectoris. Peter hält bekanntlich in seiner Theorie dieses Leidens an dem Gedanken fest, dass demselben eine Neuritis oder Neurose des Herznervengeflechtes zu Grunde liege, doch räumt er dabei, obgleich er an eine selbständige Existenz reiner Neuritiden und Neurosen glaubt, krankhafte Veränderungen in der Aorta, als direct auf das Herznervengeflecht einwirkenden Momenten, eine grosse Rolle ein. Welches nun auch immer die Ursachen der Angina pectoris sind, - mögen sie den Charakter eines Reflexes vom Magen, Darm oder von der Leber an sich tragen, oder mögen sie durch Veränderungen in der Aorta oder den Kranzarterien bedingt sein, - in allen diesen Fällen hat der diese Krankheit charakterisirende Symptomencomplex die eine oder die andere Veränderung im Herznervengeflecht zur Grundlage.

1) Clinique médicale. 1877. p. 459 u.f. - Traité elinique et pratique deș maladies du coeur. 1883. 
Von diesem Standpunkte aus findet nach Pefer die Uneinigkeit der Autoren hinsichtlich der Herzthätigkeit und des Pulscharakters bei Angina pectoris ihre Erklärung in der mehr oder weniger ausgesprochenen Affection der verschiedenen; das Herznervengeflecht bildenden Fasern.

Da nun Peter den Schwerpunkt bei Angina pectoris in das obengenannte Geflecht verlegt, so können wir auch verstehen, warum er der Schmerzhaftigkeit des 2. linken Intercostalraumes eine so hohe Bedeutung beimisst. Einmal im Besitz dieser Thatsache, ist es leicht vorauszusehen, dass obengenanntes Symptom auch in Fällen von einfachem (nicht von der Angina pectoris abhängigem) Herzklopfen gefunden werden könne. Eine solche Behauptung ist nur eine logische Schlussfolgerung aus dem Vorausgegangenen. Eine Neurose des Herznervengeflechts, welche eine Angina pectoris zur Folge hat, und eine Neurose, die blos Herzklopien bedingt, können auf diese Weise als nur verschiedene Stufen eines und desselben Prozesses angesehen werden. Und wirklich konnten wir bei fast allen neurasthenischen, hysterischen und blutarmen Individuen, welche wir untersuchten, eine mehr oder weniger ausgesprochene Schmerzhaftigkeit des 2. linken Intercostalraumes nachweisen, ja es gelang uns einigemal durch starken, aber vorsichtigen Druck auf diese Stelle eine Alteration der Herzthätigkeit im Sinne einer Veränderung des Rhythmus hervorzurufen. Ja, wir können noch weiter gehen und die Möglichkeit zugeben, dass nicht nur Neurasthenie, Hysterie und Blutarmuth eine solche Affection des Nervengeflechtes hervorrufen, sondern dass auch jede andere ïbermässige Herzaction, z. B. bei Pneumonie, bei Pleuritis oder bei acuten fieberhaften Prozessen, eine temporäre Neurose oder sogar eine Neuritis des Herznervengeflechtes zur Folge haben könne. Unsere vereinzelten Beobachtungen könnten zu Gunsten dieser Möglichkeit sprechen, und es wäre von ungemeiner Wichtigkeit, diese Thatsache auch weiterhin zu verfolgen.

Und so können wir denn bei unseren Fällen, ohne uns zur Zoit auf die Classification des eigentlichen Prozesses einzulassen, die von uns aufgefundene Schmerzhaftigkeit des 2. Intercostalraumes als eine Neurose des Herznervengeflechtes ansehen.

2) Die Schmerzhaftigkeit des linken Armnerven- 
geflechtes. Die schmerzhafte Empfindlichkeit in der linken Supraclaviculargegend und im linken Arm bildet bekanntlich eine der häufigsten Begleiterscheinungen in Anfällen von Angina pectoris. Ebenso häufig, wenn auch nicht in dem Grade, wird sie bei anderen Arten von nervösem Herzklopfen und hauptsächlich in der Graves'schen Krankheit beobachtet. Der Weg dieser Irradiation ist durch die anatomischen Verhältnisse vorgezeichnet. "Alle an der Bildung des Armnervengeflechtes theilnehmenden Nerven“, sagt Hyrtl ${ }^{1}$ ), „senden Verbindungsäste entweder zum Stamme des Sympathicus oder zum mittleren und unteren Halsganglion, der erste Brustnerv zum ersten Brustganglion." Diesen Weg nimmt auch Peter im Verein mit der Mehrzahl der Antoren an, und stellt sich damit in directen Gegensatz zu Traube ${ }^{2}$ ), welcher sich hier für einen Reflex von der inneren Herzoberfläche auf die Vasomotoren des Armes ausspricht. Ebenso mit G. Sée ${ }^{3}$ ). Letzterer erklärt, bei der Vertheidigung seiner Theorie der Angina pectoris, der Graves'schen Krankheit und and., welche darin gipfelt, dass allen diesen Prozessen ausschliesslich eine Affection des Nervus vagus zu Grunde liege, die Schmerzen im Arme als auf rein reflectorischem Wege entstandene. Nach ihm wird die Erregung der Herzfasern der Vagi dem Centrum übermittelt und irradiirt von hier aus centrifugal auf verschiedene sensible Nerven, wo sie sich dann in Form excentrischer Schmerzen äussert. Es ist hier nicht am Platze, näher auf diese Theorie einzugehen, deren antecipirte Ausschliesslichkeit keineswegs zu ihren Gunsten spricht; uns will es aber scheinen, dass die bedeutend einfachere und natürlichere Erklärung des uns hier interessirenden Symptomes auf anatomischer Grundlage mehr den Thatsachen entspricht, wie der complicirte Umweg, den Sée annimmt. Wir müssen bei der Annahme eines anatomischen Zusammenhanges unbedingt voraussetzen, dass das Armnervengeflecht bei Herzneurosen für die Uebertragung der Schmerzen auf den Arm als Zwischenglied dient. Bei der Erforschung verschiedener Fälle von Herzneurosen nach

j) Anatomiè. $\$ .836$ (russisch).

2) Gesammelte Beiträge. Th. III. S. 173.

3) Du diagnostic et du traitement des maladies du coeur. Paris 1883, p. 305 . 
dieser Richtung hin konnten wir nicht nur während des Anfalles von Herzklopfen selbst, sondern auch noch eine geraume Zeit danach eine deutlich ausgeprägte Schmerzhaftigkeit des Geflechtes selbst constatiren. Ebenso fanden wir dieselbe in vielen Fällen von Neurasthenie, gleichgültig, in welcher Form sich letztere manifestirte. Gewöhnlich beobachtet man diese Empfindlichkeit nur im linken Plexus, doch kommen nicht selten Fälle vor, wo sie sich auch auf den rechten Plexus erstreckt, obwohl in viel schwächerem Grade.

3) Die Labilität und Veränderlichkeit der Pupillen. Beard ') betrachtet diese Erscheinung als der Neurasthenie oder zum wenigsten der Nervosität angehörig. Auf die Labilität der Pupillen als ein Prodromalsymptom der tuberculösen Meningitis weist auch Steffen ${ }^{2}$ ) hin. Wenn man weiss, wie leicht erregbar das Pupillencentrum ist und eine wie grosse Anzahl von Umständen dasselbe beeinflusst [Landois $\left.\left.{ }^{5}\right)\right]$, so ist es leicht verständlich, dass a priori ein Urtheil, wodurch in unseren Fällen die Labilität bedingt wurde, schwer abgegeben werden konnte. Wir müssen indessen unbedingt hinzufügen, dass letztere in unseren Fällen ein scharf ausgeprägtes charakteristisches Symptom bildete, - das möglicher Weise nur bei ihnen allein vorkommt, - namentlich die ausserordentliche Schnelligkeit, mit welcher der Durchmesser unter sonst gleichen Bedingungen wechselte.

4) Die gesteigerten Muskel- und Sehnenreflexe, auf welche Immermann in seinem Aufsatz über Blutarmuth hinweist, ebenso wie Beard (1. c.), haben wir in fast allen Fällen von Neurasthenie, hauptsächlich in der cerebrospinalen und auch in allen mehr oder weniger ausgeprägten Formen, wie Blutarmuth und Bleichsucht, constatirt. Nicht immer ist diese Steigerung auf beiden Seiten gleichmässig ausgebildet. In weitaus den meisten Fällen wird die eine Seite, meist die linke, mehr ergriffen. Durch leichte Schläge mit dem Percussionshammer kann man zeitweilig (natürlich müssen sich die Muskeln im erschlafften

1) Die Nervenschwäche. 1881. S. 27.

2) Gerhardt, Handbuch der Kinderkrankheiten. 1880. Th. V. Abth. 1. 2. Hälfte. S. 454.

3) Landois, Physiologie. 1880. S. 798. 799, 
Zustande befinden) äusserst starke Contractionen der Schulter und Vorderarmmuskeln hervorrufen, und in mehr ausgesprochenen Fällen erhält man an den Muskeln des Thenar und Hypothenar, wie an denen der Brust das gleiche Phänomen. Nicht selten kommt ferner nach einem leichten Schlage ein tetanischer oder klonischer Krampf der ganzen Extremität zur Beobachtung.

In einem Falle erfolgten bei einer unserer Kranken, die an ausgeprägter cerebrospinaler Neurasthenie litt, nach einem Schlag auf den M. supinator longus jed esmal starke klonische Krämpfe der ganzen entsprechenden Körperhäfte. Dasselbe können wir hinsichtlich der Sehnenreflexe, insbesondere des Patellarreflexes, constatiren. Parallel dieser übergrossen Reizempfindlichkeit der Musculatur geht gewöhnlich auch diejenige des vasomotorischen Systems, die sich in einem schnellen Wechsel von Röthe und Blässe des Gesichtes, wie in localen Asphyxien, Ohnmachten etc. bekundet.

5) Herzklopfen und Arhythmie des Herzens gehören in mehreren Fällen nicht zu den constanten Symptomen, sondern treten nur zeitweise als Begleiterscheinungen eines Fieberanfalls auf. Mit dem Abfalle der Temperatur schwand auch die Erregt. heit des Herzens. Bei beiden Patientinnen bot das Herz keine Abweichung von der Norm dar; beide Durchmesser blieben unverändert, die Töne waren rein, Geräusche nicht vorhanden. Das Einzige, was sich constatiren liess, war eine energischere und frequentere Contraction und ein leicht metallisches Timbre des ersten Tones an der Herzspitze, also dasselbe, was die Franzosen "cliquetis metallique" nennen. Der Puls war bei beiden Kranken während der Anfälle beschleunigt, bis 130 in der Minute, klein und fadenförmig. Unabhängig davon dürfte noch die Thatsache von Interesse sein, dass während der freien Intervalle beide Patientinnen durchaus keine erhöhte Reizbarkeit zeigten, wenigstens unter alltäglichen Verhältnissen. Beide gingen leicht und rasch, und das Treppensteigen selbst bis in die 5. Etage ging ohne, jede Athembeschwerden vor sich.

Anders aber reagirte das Herz auf psychische Einflüsse. Das, was sogar durch anhaltendes Laufen nicht hervorgerufen werden konnte, bewirkte die kleinste Ueberraschung, die mindeste Aufregung. Es konnte folglich keinem $Z_{\text {weifel }}$ unterliegen, 
dass wir es hier mit einer Neurose zu thun hatten. Es war dabei nur fraglich, ob dieselbe eine primäre oder eine secundäre sei, und welcher Nervenapparat namentlich sich an dem Prozesse betheiligte.

Eine beschleunigte Herzthätigkeit kann bekanntlich hervorgerufen werden: a) durch eine Reizung der automatischen Herzganglien, b) durch eine Affection der regulatorischen Vagusthätigkeit, oder genauer durch eine Paralyse dieses Nerven, c) durch eine Affection der beschleunigenden Fasern und endlich d) auf rein vasomotorischem Wege.

Um in diesen 2 za besprechenden Fällen einen Reiz der automatischen Centren zuzulassen, erscheint es nothwendig, das Vorhandensein eines Reizerzengers im Herzen selbst anzunehmen. Analog den Experimenten von Landois ${ }^{1}$ ), könnten als solche Reizerzeuger Aortenfehler, atheromatöse Prozesse, Ischämie in Folge von Stenose der Kranzarterien, Krankheiten des Myocardiums u. ähnl. angesehen werden (Eulenburg), in unseren Fällen aber fehlten derartige Ursachen.

Um die Theilnahme des N. vagus an der Beschleunigung der Herzthätigkeit zuzugeben, müsste man eine temporäre Paralyse desselben annehmen, welche zuweilen ziemlich lange anhalten und sich wiederholen kann, und dabei einzig und allein unter dem Einflusse psychischer Affecte. - Es bleibt nichts weiter übrig, als entweder eine Reizung der Acceleratoren oder einen rein vasomotorischen Weg anzunehmen. Für die erste Annahme sprach bei beiden Patienten die Empfindlichkeit des zweiten linken Intercostalraumes und des anliegenden Theiles des Sternums, da dieser Stelle die Lage des Plexus cardiacus entspricht und in ihm sich alle von verschiedenen Seiten kommenden sympathischen Nerven des Herzens vereinigen (Lancereaux, Peter, Eulenburg). Ein rein vasomotorisches Moment für die Erklärung der beschleunigten Herzthätigkeit könnte man zulassen nach Analogie der Angina pectoris vasomotoria, welche von Landois schon im Jahre 1866 beschrieben wurde. Es ist bekannt, dass die Reizung der Vasomotoren den Blutdruck

I) Die directe Herzreizung. Greifswalder med. Beiträge. II. 1864. S. 161 und Lehrb. d. Physiol. d. Menschen. S. 109.1880. 
durch Verengerung der Gefässe bedeutend erhöht. Diese Erhöhung des Blutdruckes wirkt unmittelbar als mechanischer Reiz auf den linken Ventrikel und verursacht eine Verstärkung und Beschleunigung (Heidenhain, Slavjansky) der schon ohnehin, in Folge der Gefässverengung, beschleunigten Herzthätigkeit.

Wir lassen es unentschieden, welches von den beiden letzteren Momenten in unseren Fällen die Ursache war. Möglich ist es, dass beide mitgewirkt haben. Wir können übrigens mit einigem Recht annehmen, dass wir in vielen Anfällen es nur mit dem letzteren Momente zu thun hatten, d. h. mit dem vasomotorischen Effect, denn dafür spricht wenigstens die Stärke des Herzstosses und der metallische Beiklang beim Auscultiren. Wir wiederholen, wir lassen diese Frage unentschieden, um so mehr, da auch das physiologische Experiment uns lehrt, dass bei der Reizung des Centrums der Acceleratoren die Betheiligung des vasomotorischen Centrums sich schwer beseitigen lässt. Wir lassen uns daher in keine weiteren Details ein und nehmen für unsere Fälle die Möglichkeit der Wirkung beider Momente an. Eine solche Erklärung erscheint uns am passendsten, da sie nicht blos am besten den Charakter der Herzthätigkeit erklärt, sondern auch mit anderen Erscheinungen übereinstimmt. Denn wenn wir es im gegebenen Falle mit im Herzen selbst liegenden Ursachen zu thun hätten, so würde es unbegreiflich erscheinen, warum psychische Einflüsse hauptsächlich Herzklopfen verursachten; es liesse sich auch schwer eine Erklärung für die zuweilen sehr langen freien Perioden finden. Gegen eine selbständige Neurose des Plexus cardiacus im Sinne Peter's spricht ferner noch der Umstand, dass das Herzklopfen-in unseren Fällen niemals allein, sondern immer mit gleichzeitiger Temperaturerhöhung auftrat. Wenn wir uns ferner erinnern, dass wir bereits früher die Besprechung verstärkter Muskel- und Sehnenreflexe, das Vorhandensein einer erhöhten reflectorischen Rejzbarkeit des Rückenmarkes zugelassen haben, so wird die Reizbarkeit des N. sympathicus und speciell des vasomotorischen Centrums schon durch die Analogie allein gegeben sein.

Unabhängig von den 5 so eben analysirten, für unsere beiden Kranken gemeinsamen Symptomen, wurden noch bei der ersten (Fall II) beobachtet: 1) Erscheinungen von Seiten des Magens, 
2) Salivation und 3) Schweisse, - bei der zweiten (Fall III) Husten.

In Anbetracht dessen, dass bei der ersten Kranken die Erscheinungen von Seiten des Magens zu dem gewöhnlich normalen Zustande der Verdauungswege in directem Widerspruch standen, dass diese Erscheinungen nur während der Anfälle auftraten und mit deren Ende verschwanden, dass das von Zeit zu Zeit sich einstellende Erbrechen fast ausschliesslich durch verticale Lage bedingt wurde, wenn wir ferner auch die Salivation berücksichtigen, - ist es klar, dass die Hauptursache dieser Erscheinungen nicht in den Verdauungswegen lag. Der ganze Complex nervöser Erscheinungen zwingt uns, das in Rede stehende Symptom zur Kategorie derjenigen nervösen Dyspepsien und Enteropathien zu rechnen, die wir in einem unserer früheren Aufsätze besprochen haben ${ }^{1}$ ). Damals stellten wir diese Erscheinungen in directe Abhängigkeit von einer Neurasthenie; hier möchten wir aber noch darauf hinweisen, dass in den jetzt zu besprechenden Fällen eine Bedingung vorhanden ist, welche unabhängig von einer Neurasthenie den Schlüssel zur Aufklärung geben kann. Wir meinen das Herzklopfen. Der Zusammenhang der Symptome zwischen Herz und Magen ist bereits seit lange anerkannt. Von diesem Zusammenhange sprach schon Corvisart ${ }^{2}$ ) und in newerer Zeit I eared ${ }^{3}$ ), Vulpian ${ }^{4}$, Potain ${ }^{5}$ ), Trousseau ${ }^{6}$ ) u. A.

Indem wir die Erscheinungen von Seiten des Magens bei unserer Kranken (Taf. II) durch gestörte Innervation des Darmkanals erklären und dieselbe Form der Gleichgewichtsstörung annehmen, welche wir in unserer erwähnten Arbeit zugelassen haben, - glauben wir, dass diese Erscheinungen erklärt werden können, wenn wir von den Gesichtspunkten der Experimente Basch's ausgehen, welcher die Ursache der peristaltischen Be-

1) Zur Pathologie der Neurosen des Darmkanals. „Wratsch“ 1882. und Revue de médecine. 1883. No. 10 u. 12.

3) Essai sur les maladies et les lésions organiques du coeur. 1811. 2. Aufl. p. 134 .

3) On disguised diseases of the heart. Med. Times and Gaz. 1867. Vol. I. p. 695 .

4) Des synergies morbides. Gaz. méd. de Paris. 1879. p. 83 u. ff.

5) Clinique médicale de l'Hôtel-Dieu.

6) Sitzungsber. d. Akad. der Wissenschaften. Wien 1874. S. 7 u. ff. 
wegungen in den vasomotorischen Eigenschaften des N. splanchnicus sieht. Die Reizung des peripherischen Endes dieses Nerven ruft, nach Basch, eine Verengerung aller Gefässe nicht nuir des Mesenteriums, sondern auch der Darmwände mit nachfolgender Erweiterung (Diastole) hervor. Diese Diastole fält übrigens nicht immer mit dem Erschlaffungszustande zusammen. Zuweilen tritt nach der Reizung ein solcher Zustand ein, wo in Folge des Aufhörens der Contraction der Längsmuskeln die Gedärme in einer Richtung ganz unbeweglich bleiben, während die Ringmuskeln tonisch contrahirt sind. Höchst selten wird ausserdem beobachtet, dass diese allgemeine tonische Contraction der Ringmuskeln partiellen Abschnürungen weicht.

Diese Thatsachen erklären in Vielem den Charakter der Darmneurosen und geben zugleich den Schlüssel zur Aufklärung der Erscheinungen von Seiten des Magens bei unserer Kranken; da sowohl der eine, als der andere Zustand des $\mathrm{N}$. splanchnicus eine Verengerung oder Erweiterung der Gefässe des Darmkanals hervorruft, die Peristaltik schwächt oder beschleunigt und folglich Verstopfung oder Durchfall zur Folge.hat. Von diesem Gesichtspunkte aus muss man die Erscheinungen von Seiten des Magens auch bei dieser Kranken als Erscheinungen vasomotorischen Charakters bezeichnen.

Die Salivation bei unserer zweiten Kranken (Fall II) zeichnete sich dadurch aus, dass der Speichel besonders klebrig und fadenziehend war, so dass das Kind über „Fäden im Munde“ klagte. Solch ein klebriger Speichel wird nur bei Reizung des N. sympathicus beobachtet (Landois 1. c. S. 267).

Was den Schweiss anbetrifft, so bedarf sein vasomotorischer Charakter keines weiteren Beweises.

Der bei der dritten Kranken (Fall III) beobachtete Husten begleitete alle Anfälle und bot stets denselben, schon früher beschriebenen Charakter dar. Das Erscheinen des Hustens bei hoher Temperatur brachte uns, beim Fehlen fester Anhaltspunkte für die Diagnose, auf den Gedanken, einen Lungenprozess anzunehmen. Als wiederholte Untersuchungen ein durchaus negatives Resultat ergaben, so blieb dennoch der Zweifel bestehen, ob nicht der Prozess in den Bronchialdrüsen niste, wenngleich der Zustand des Lymphsystems kein Recht zu einer solchen Ver- 
muthung gab. Erst später, als der Zusammenhang zwischen Husten, Herzklopfen und Temperatur und die Abhängigkeit des ersteren von den letzteren deutlich hervortrat, konnte man über das Wesen und die Ursache des Hustens klarer werden. Wir hatten es offenbar nur mit einer Reizung im Gebiet des N. laryngeus sup: zu thun. Die weiteren Folgerungen in Bezug auf die Ausgangspunkte dieser Reizung konnten verschieden sein. Man konnte z. B. annehmen, dass die, durch irgend welche Veränderungen im N. sympathicus oder in den sympathischen Fasern des Plexus cardiacus verursachte erhöhte Herzthätigkeit bei mehr oder weniger längerer Dauer consecutiv einen Reiz auf die peripherischen Verzweigungen des $N$. vagus ausüben und sich von hier centripetal auf den N. laryngeus sup. fortpflanzen konnte; oder man konnte centrale vasomotorische Ursachen zulassen, da der N. laryngeus sup. seine vasomotorischen Fäden direct aus dem Ganglion cervicale sup. n. sympathici entnimmt; oder endlich man konnte diesen Husten als Folge der Reizung des Hustencentrums [Kohts $]^{1}$ ) ansehen. In letzterer Beziehung könnten wir, als auf əin eclatantes Beispiel, auf die Ataxie des Kehlkopfes ${ }^{2}$ ) hinweisen, bei welcher die Ursache der Erscheinungen im verlängerten Mark liegt und, nach den. klinischen Erscheinungen zu urtheilen, nicht immer eine materielle Grundlage hat.

Um mit der Besprechung der Fälle II und III zu schliessen, bleibt uns noch äbrig, das Hauptsymptom, - die Temperaturerhöhung, zu erklären. In beiden Fällen stellt sie sich in den Vordergrund: das Krankheitsbild beginnt mit der Temperatursteigerung und durch sie wird die Stärke und Dauer des Anfalles bedingt. Schon aus dem Umstande, dass alle folgenden Anfälle auf rein nervösem Wege zu erklären sind, konnten wir a priori den Schluss ziehen, dass auch die Temperatur zur Kategorie der nervösen Erscheinungen gehöre. Abgesehen jedoch von diesem indirecten Beweis, bietet uns die Aetiologie jedes einzelnen Anfalles auch einen directen Beweis. Es war ohne Zweifel, und wir konnten es mehr als einmal genau verfolgen, dass die Temperaturerhöhung sofort den psychischen Affecten

1) Ich citire nach Landois.

2) Siehe meinen Aufsatz "Die Affectionen des Larynx bei Ataxie". "Wratsch" 1881. und Revue de médecine 1881. 
folgte. Diese Thatsache findet ibre Bestätigung in der Literatur. So spricht noch im Jahre 1867 Botkin ${ }^{1}$ ) über Temperaturerhöhungen bei Katheterisation und unter dem Einflusse psychischer Ursachen und indem er den ersten Umstand durch Veränderungen der Thätigkeit der regulirenden Centren und den Einfluss der Reizung sympathischer Nerven erklärt, bemerkt er behufs des zweiten Umstandes: „dass dieser Umstand uns gestattet eine Reizung des Wärmecentrums auch auf dem Wege durch das Grosshirn anzunehmen". (Charcot ${ }^{2}$ ) weist auf die Möglichkeit sehr hoher Temperaturen bei Hysteroepilepsie und sog. attaques epileptiformes bei allgemeiner Paralyse und bei multipler Sclerose hin.

Diese Literaturangaben im Verein mit der Thatsache, dass in unseren Fällen die gebräuchlichen Antipyretica die Temperatur nicht herabsetzen konnten, während dagegen Bromkalium, Champagner und Sauerstoff die Temperatur rasch zum Sinken brachten, gestatten die Annahme, dass die Temperaturerhöhung in unseren 2 Fällen centralen Ursprunges oder richtiger durch Functionsstörung des vasomotorischen Centrums bedingt sei, dessen Theilnahme in den vorliegenden Fällen schon ohnehin deutlich hervortritt. Die Wirkung des Sauerstoffs scheint hier, wie auch im ersten Falle, die bereits von uns angefübrten Combinationen zu bestätigen. Wohl konnte hier von allgemeiner Anämie als bedingendem Moment keine Rede sein; aber zu Gunsten einer localen, durch deatliche cerebrale Neurasthenie bedingten Gehirnanämie sprachen nicht wenige Umstände. Die Bedeutung einer solchen Anämie für das vasomotorische Centrum erhellt aus den Experimenten Navalichin's ${ }^{3}$ ).

Fall IV. Bei der Besprechung dieses Falles müssen wir vor Allem auf die Identität vieler Symptome mit denjenigen der 2 vorherbesprochenen Fälle, d. h. des zweiten und dritten, besonders des dritten hinweisen. Um Wiederholungen zu vermeiden, wollen wir das allen Fällen Gemeinsame stillschweigend. übergehen, indem wir uns dabei auf die bereits angeführten Erklärungen berufen und blos diejenigen Erscheinungen berücksichtigen, welche nur dieser Kranken eigen waren.

1) Cursus der Klinik für innere Krankheiten. Heft II. S. 26.

2) Leçons sur les maladies du système nerveux. Bd.I. p.376.

3) Ich citire nach Landois. 
Die Anamnese und der Untersuchungsbefund liessen keinen Zweifel darüber aufkommen, dass wir es mit Chlorose zu thun hatten. Das Bestehen der Chlorose datirte offenbar schon seit lange und bekundete ein besonderes Bestreben sich in der centralen Sphäre zu localisiren, worauf die heftigen Kopfschmerzen und die in der Anamnese notirten Gehirnerscheinungen hinwiesen. "Die reizbare Schwäche" des Nervensystems, die gewöhnliche Begleiterin der Chlorose, welche Immermann hauptsächlich dem Mangel an Hämoglobin zuschreibt, erklärte zur Genüge die meisten vom Nervensystem aus bedingten Erscheinungen. Im weiteren Verlauf traten jedoch Erscheinungen auf, welche, wie wir bereits erwähnt haben, nicht wenig die Diagnose erschwerten; in dieser Beziehung weisen wir vor Allem auf die Temperatur hin. Fiebertemperaturen gehören bekanntlich nicht za den Symptomen der Chlorose; nach Immermann ${ }^{3}$ bilden solche mit Fieber schon eine Uebergangsstufe zur perniciösen Anämie. Mit Berücksichtigung dessen war es bei dem gegebenen Charakter und der Höhe der Temperatur, bei gleichzeitigem, hartnäckigem, quälenden Husten, einem systolischen Geräusch an der Herzspitze und Oedem der Füsse natürlich, an das Vorhandensein entweder 1) eines Lungenprozesses, oder 2) einer Endocarditis, oder 3) einer Venenthrombose, Phlebitis oder 4) perniciöser Anämie zu denken.

1) Wenn auch der hartnäckige, quälende Husten sich durch Momente erklären liess, über welche wir bereits im 3 . Falle gesprochen haben (eine durch die Reizbarkeit des Herzens bedingte Reizung im Gebiet des N. laryngeus sup.), so konnte doch andererseits die Stärke des Hustens in Verbindung mit einem solchen ätiologischen Momente, wie Chlorose, den Gedanken an ein ernstes Lungenleiden aufkommen lassen. Schon längst ist die grosse ätiologische Bedeutung der Anämie und Chlorose bei Lungenphthise bekannt, und Fälle, wo die letztere durch die erstere maskirt wird, sind nicht selten. Diese Thatsachen bestimmten Trousseau und Immermann, in solchen Fällen äusserste Vorsicht zu empfehlen; der letztere weist u. a. auf die Temperatur als ein wichtiges Kriterium hin. In unserem Falle

1) Ziemssen's Handb. d. spec. Pathologie. Bd.XIII. S. 569. Ausg. 1875. 
jedoch war es gerade die Temperatur, welche Schwierigkeiten verursachte. Die wiederholt vorgenommenen, äusserst genauen Untersuchungen der Lungen führten indessen zu keinem Resultate, so dass die Frage nach der Theilnahme der Lungen am Prozesse damit hinfällig wurde.

2) So lange die Geräusche an der Herzbasis und in den Halsgefässen gehört wurden, konnten sie vollkommen durch Chlorose erklärt werden. Das Erscheinen jedoch eines systolischen Geräusches an der Herzspitze schloss zwar eine Chlorose nicht aus, sprach aber bei gleichzeitiger Temperaturerhöhung, Vergrösserung, wenn auch temporärer, des Querdurchmessers des Herzens und Schüttelfrösten zugleich für die Möglichkeit einer Endocarditis. Auf die Disposition chlorotischer Kranken zu Erkrankungen des Endocardiums hat bereits Virchow hingewiesen, wobei er diesen Umstand mit der von ihm bewiesenen Anomalie der Gefässe bei solchen Kranken in Zusammenhang bringt. Das Auftreten des Geräusches an der Herzspitze nach dem Erscheinen der Basalgeräusche, seine äusserste Weichheit und Schwäche, seine Veränderlichkeit im erregten und ruhigen Zustande bei gleichzeitigem Fehlen einer stabilen Veränderung des Querdurchmessers des Herzens sprachen gegen Endocarditis. Das Fehlen eines typhösen Zustandes and einer Milzvergrösserung schlossen auch eine Endocarditis ulcerosa aus, auf welche Temperatur und Schüttelfröste hätten hinweisen können.

3) Das Zusammenfallen der höchsten Temperatur mit dem Oedem der Füsse konnte den Gedanken wachrufen, ob nicht überhaupt die Temperatur in diesem Falle von einem Prozesse an den Füssen abhängig sei. Eine sorgfältige chirurgische Untersuchung jedoch ergab weder an der einen, noch an der anderen Extremität irgend welche Anzeichen von Phlebitis oder Lymphangioitis. Andererseits aber liessen das schnelle Anschwellen des rechten Oberschenkels und der Charakter der Geschwulst zuerst eine Venenthrombose voraussetzen; dafür sprach ebenfalls ein höchst wichtiger Umstand in der Anamnese - die bereits im Jahre 1877 entstandene Venenthrombose dieser Extremität. Damals trat dieselbe nach einer schweren Lungenentzündung auf, die sich erst am 14. Tage löste. Indem wir die Entstehungsursachen der Thrombose damals und jetzt vergleichen, finden wir 
einen sehr wichtigen gemeinsamen $\mathrm{Zug}$. Eine jede, selbst die leichteste Pneumonie, steigert die Arbeit des Herzens sehr bedeutend, und um so mehr eine solche, welche 14 Tage anhält. Offenbar war die Herzthätigkeit zu Ende dieser Krankheit sehr geschwächt, die vis a tergo genügte nicht mehr. Auch hier finden wir dieselben Bedingungen, nur unter dem Einflusse anderer Ursachen. Eine lange Periode der Gereiztheit des Herzens, die dem Erkranken der Füsse voranging, führte schliesslich zu derselben ungenügenden Herzthätigkeit, was sich auch durch die Ueberfüllung des rechten Herzens zu erkennen gab, und eine, wenn auch temporäre Vergrösserung des Querdurchmessers zur Folge hatte. Die Ursachen waren auf diese Weise in beiden Fällen offenbar dieselben, sie konnten jedoch nur eine sog. marantische Thrombose bedingen, auf deren mögliches Vorkommen bei Anämischen auch Immermann (I. c. S. 399) hinweist. In Anbetracht dieser Combinationen sehen wir keinen Grund, unter den nämlichen Bedingungen nicht auch die nämliche Form der Thrombose des linken Oberschenkels zuzulassen. Es ist allerdings wahr, ein Unterschied zwischen beiden Thrombosen offenbarte sich in der Dauer; wenn wir jedoch bedenken, dass die zweite einen schon vorbereiteten Boden (durch vorhergegangene Thrombose) für eine schnellere Entstehung der Collateralwege vorfand, so erscheint es begreiflich, warum der Prozess in der rechten Extremität bedeutend schneller seinen Abschluss fand.

Wenn nun derselbe für eine Thrombose erklärt werden musste, so kann er wohl kaum als Grund einer so hohen Temperatur angenommen werden, um so weniger, als bei der zweiten Thrombose die Temperatur sank, bevor sich noch die Geschwulst vollkommen entwickelt hatte, wie dies aus der Curve ersichtbar ist. So kann auch von dieser Seite her die Temperatur nicht erklärt werden.

4) Die innige Verwandtschaft zwischen hochgradiger Chlorose und perniciöser Anämie drängte nun die Frage auf, ob wir es im gegebenen Falle nicht mit der letzteren Krankheit zu thun hätten.

Immermann bezeichnet mit dem Namen perniciöse Anämie diejenigen Fälle hochgradiger Anämie, welche unvermeidlich und schnell, trotz jeder Behandlung, zum Tode führen. 
Von diesem Gesichtspunkte aus sieht er den Unterschied dieser Form von der Chlorose darin, dass letztere nicht so rasch fortschreitet und weniger perniciös ist und dass die Behandlung dabei oft Erfolge aufweist. Indem Biermer und Immermann das gemeinsame Bild der Anfangsstadien der perniciösen Anämie liefern, welches vollkommen identisch ist mit dem Krankheitsbilde der Chlorose, weisen sie auf folgende Hauptsymptome hin: 1) ein systolisches Geräusch an der Herzspitze und Basis und Geräusche in den Halsvenen, welche den Geräuschen bei Anämie und Chlorose vollkommen gleichen und sich von den letztèren nur durch grössere Beständigkeit und Stärke unterscheiden. 2) Das Fieber, welches alle Fälle ohne Ausnahme begleitet und meistentheils erst gegen Ende sich offenbart und 3) Blutextravasate, als eine weniger häufige Erscheinung. Wenn wir nun diesen Symptomencomplex bei der Analyse unseres Falles vor Augen behalten und dabei die Blutextravasate, als eine relativ seltene Erscheinung unberücksichtigt lassen, müssten wir unseren Fall unbedingt als Anaemia perniciosa bezeichnen, wenn dieser Diagnose die Genesung nicht widersprechen würde. Wenn aber in Folge der Genesung unser Fall nicht hierher gehört, so muss er offenbar den mit Fieber verbundenen Formen der Chlorose zugezählt werden, welche Immermann für Uebergangsstufen zwischen dieser und der perniciösen Anämie ansieht, indem er dabei, wenn man sich so ausdrücken kann, blos einen quantitativen Unterschied gelten lässt. Untersuchen wie nun, wie sich die Temperatur in entwickelten Fällen von perniciöser Anämie erklären lässt.

Im Anschlusse an Biermer nimmt Immermann einen "humoralen" Grund des Fiebers an, d. h. er stellt das Fieber in directe Abhängigkejt von der Qualität des Blutes; legt sich aber zugleich die Frage vor, auf welche Weise die Anämie, und zwar eine solche, welche von Verminderung des Hämoglobins begleitet ist, Fiebertemperaturen erzeugen kann, da es doch natïrlicher wäre ein Sinken der Temperatur zu erwarten. Indem der Autor, wegen der Neuheit der Frage und aus Mangel an Daten davon absteht, eine directe Antwort auf diese Frage zu geben, zeichnet er blos die allgemeinen Ziige und bleibt auf dem Wege indirecter Combinationen vor der Hand bei folgender Erklärung stehen: 
"das Fieber erscheint dann, wenn die Quantität der rothen Blutkörperchen in Folge irgend welcher Ursachen unter eine gewisse, jedenfalls sehr niedrige Norm fällt". Als einen empirisehen $\mathrm{Be}$ weis sieht dieser Autor die bekannten Beobachtungen Frese's an, denen zufolge eine ausgiebige Venaesection nach einem bedeutenden, jedoch kurzen Sinken ein Steigen der Temperatur verursacht; ferner die Thatsache, dass auch bei hochgradiger Chlorose zuweilen Fieber beobachtet wird und dass endlich anämische Reconvalescenten sehr zu Temperaturschwankungen geneigt sind. Indem sich Immermann weiter darüber ausspricht, auf welche Weise hochgradige Oligocythämie eine hohe Temperatur bedingen könne, hält er, da keine Aussicht vorhanden, letztere durch verstärlkte Metamorphose zu erklären, für wahrscheinlicher, dass dem Fieber eine Nekrobiose der Gewebe zu Grunde liege.

Die Unzulänglichkeit dieser Voraussetzung ist offenbar. Denn wenn wir uns mit derselben begnügen wollten, so müssten wir die Gesetzmässigkeit einer Nekrobiose mit allen ihren Consequenzen auch bei jeder anderen Oligocythämie zulassen; dann aber würde der Umstand, dass die Oligämie mit einem Sinken der Temperatur verbunden ist, vollkommen unbegreiflich erscheinen. Es können jedoch, ganz unabhängig davon, auch die empirischen Beweise Immermann's wohl kaum als hinreichend anerkannt werden. Seinen Erklärungen gemäss müssten wir nehmlich annehmen, dass die durch profuse Venaesection bedingte Temperaturerhöhung von einer Nekrobiose abhängt und dass den leichten Temperaturschwankungen bei anämischen Reconvalescenten und der Temperaturerhöhung unter dem Einflusse psychischer Affecte auch eine Nekrobiose zu Grunde liege. Wenn er sich jedoch auf febrile Chlorose beruft, so erscheint dieser Umstand sehr wenig beweisend, da er selbst diese Fälle als niedrigste Grade einer perniciösen Anämie ansieht. Offenbar liegt also nicht hierin der Grund des Fiebers; höchst wahrscheinlich auch nicht in den "humoralen" Eigenschaften des Blutes, denn nach Immermann selbst zeigt weder die mikroskopische Untersuchung des Blutes, noch das quantitative Verhältniss der rothen Blutkörperchen zu den weissen irgend eine Abweichung (Biermer). Von dieser Seite her können wir daher keinen Aufschluss 
erwarten. Sehen wir daher, ob wir nicht von einem anderen Standpunkte aus eine Erklärung erzielen können, selbst wenn wir riskiren, eine "überflüssige" Theorie aufzubauen. Wir begründen unseren Versuch auf Daten und Combinationen, die wir aus den 3 ersten Krankengeschichten gewinnen, deren Identität in Bezug auf Symptome, mit der vierten wohl kaum neuer Beweise bedarf.

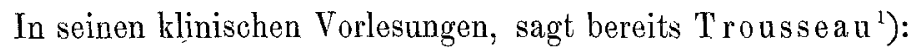
„meiner Meinung nach gehört die Chlorose eher zu den Nervenkrankheiten" und weist auf eine Reihe nervöser Erscheinungen hin, von welchen jede irgend wie ausgeprägte Chlorose begleitet wird. In den nervösen Functionsstörungen sieht er den Grund bedeutender Ernährungsveränderungen, und sich auf physiologische Experimente berufend, weist er auf die Möglichkeit auch qualitativer Veränderungen des Blutes unter dem Einflusse gestörter Innervation hin (Tarchanow, die Innervation der Milz). Die Innervationsstörung der Lungen, Leber, Milz u. s. w. kann nicht ohne bedeutenden Einfluss auf die Qualität des Blutes bleiben. Wenn wir nun zu unserem Falle zurückkehren, sind wir dann nicht berechtigt, ihn der von Trousseau gegebenen Definition der Chlorose gemäss zu erklären? Schon das einfache Aufzählen der Symptome weist direct auf die nervöse Natur des Prozesses hin. Dafür spricht ebenfalls die fast völlige Identität mit dem zweiten und dritten Falle. Von diesem Gesichtspunkte aus und unter Zuhülfenabme der für die vorhergehenden Fälle angenommenen Erklärungen sind wir im Stande, die hohe Temperatur als eine auf rein centralem Wege entstandene aufzufassen. Und in der That, wenn wir die anamnestischen Angaben im Auge behalten, nehmlich heftige Kopfschmerzen (offenbar vasomotorischen Charakters), die Reizbarkeit des Herzens, die Veränderlichkeit seines Querdurchmessers, den hohen Grad reflectorischer Reizbarkeit des Rückenmarkes und eine Reibe anderer Erscheinungen (den schnellen Wechsel der Röthe und Blässe des Gesichts, die Kälte der Extremitäten), welche direct auf eine Innervationsstörung der Vasomotoren hinweisen, können wir, kraft der Analogie, einen Schritt vorwärts machen und zugeben, dass die hohe

1) Clinique médicale de l'Hôtel-Dieu. T. III. p. 514. Ausgabe 1882. 
Temperatur das Resultat von Veränderungen im vasomotorischen Centrum ist. Dazu bedarf es blos einer gewissen Verstärkung der schon ohnehin vorhandenen anomalen Thätigkeit der Vasomotoren. Ferner können wir von diesem Gesichtspunkte aus die Temperaturerhöhung nach Blutentziehungen und die leichten Temperaturschwankangen bei anämischen Reconvalescenten erklären, - Beispiele, welche Immermann anführt zum Beweise seiner "humoralen Anschauung" des Fiebers bei perniciöser Anämie. Zu Gunsten dieser Erklärung des Fiebers nach Aderlässen sprechen auch die Untersuchungen des Prof. J. T. Tschudnowsky ${ }^{1}$ ), welche Immermann offenbar ganz ausser Acht gelassen hat. Nachdem Tschudnowsky bewiesen, dass die Temperaturerhöhung nicht durch Veränderungen der Intensivität chemischer Prozesse im Körper bedingt wird, kommt er zu folgenden Schlüssen: 1) „unter dem Einflusse des Aderlasses vermindert sich das Volumen der Haut- und Lungengefässe, die Anzahl der Austauschpunkte zwischen der Wärme des Blutes und der umgebenden Atmosphäre nimmt ab, daraus resultirt die Temperaturerhöhung"; 2) da die Anzahl der Austauschpunkte zwischen dem Blute und den umgebenden Medien abnimmt und da ferner der Aderlass den Eintritt von Wasser aus den umgebenden Geweben in das Blut bedingt, ist es klar, dass "der Aderlass ein verringertes Verdansten des Wassers an der Oberfläche bedingt, das verringerte Verdunsten aber hat eine Steigerung der Körpertemperatur zur Folge". Wenn nun die Temperaturerhöhung nach Blutentziehungen von der regulatorischen Thätigkeit der Hautgefässe abbängig gemacht wird, so widerspricht dieser Umstand direct dem Schlusse, welchen Immermann aus den Versuchen Fresé's zieht, sowie seiner "humoralen" Anschauung, und kann mit grösserem Recht für eine Bestätigung unserer Anschauungsweise gelten.

Wenn wir nun die hohe Temperatur bei unserer vierten Kranken durch Functionsstörungen des vasomotorischen Centrums erklären können und wenn wir ferner den Umstand in Betracht ziehen, dass, nach Immermann, die perniciöse Anämie nur den

1) J. Tschudnowsky, Material zum klinischen Studium der Wirkung des Aderlasses. Diss. St. Petersburg 1869. 
höchsten Grad der Chlorose darstellt und sich von der letzteren nur dureh den Ausgang unterscheidet, - könnten wir dann nicht die hohe Temperatur bei perniciöser Anämie ebenfalls als eine durch etwaige materielle Veränderungen im vasomotorischen Centrum bedingte ansehen? mit anderen Worten könnten wir nicht zugeben, dass die perniciöse Anämie eine Neurose ist, äbnlich den 2 anderen von der Klinik bereits anerkannten Neurosen, - der Graves'schen und Addison'schen Krankheit? Und sprechen nicht die absolut negativen Daten aus der pathologischen Anatomie zu Gunsten dieser Annahme? Dieser Absolutismus ist übrigens relativ, denn um ihn zuzulassen, bedürfen wir mikroskopischer Untersuchungen der Nervencentren.

Hiermit möge die Analyse unserer Krankheitsgeschichte beendet sein. Halten wir uns nun die Resultate derselben vor, so ersehen wir daraus, dass, abgesehen von den durch die Krankheitsbilder der Diphtherie, Neurasthenie und Bleichsucht bedingten Unterschieden, die Ursache der allen 4 Fällen gemeinsamen Temperaturerhöhung als die gleiche angesehen werden muss; denn in dem Lichte unserer Beurtheilung erseheint der fieberhafte Zustand bei allen Kranken als Folge centraler Ursachen, welche die regulatorische Thätigkeit des vasomotorischen Centrums beeinträchtigt haben. Die Frage, auf welche Weise das letztere erregt wird, ob direct oder durch Vermittelung anderer in die Hirnsubstanz verlegter Centren (Tschetschichin ${ }^{1}$ ), Naunyn ${ }^{2}$ ) und Quincke - regulatorisches Centram; Eulenburg und Landois ${ }^{3}$ ) - Centrum in der Rindensubstanz) könnte, wenigstens nach unseren Fällen zu urtheilen, sowohl in dem einen wie im anderen Sinne bejahend entschieden werden.

Indem wir somit glauben, eine Innervationsaffection des vasomotorischen Centrums annehmen und sie als unmittelbare Ursache der thermischen Veränderungen in unseren Fällen ansehen $z u$ müssen, sind wir weit davon entfernt, physiologische Gesetze entscheiden zu wollen, und wünschen uns nur dahin verstanden zu wissen, dass, wie beschaffen auch immer die Centren der Wärmeregulation sein mögen und wohin man sie auch ver-

1) Arch. f. Anat. u. Physiol. 1866. S. 151.

2) Arch. f. Anat. u. Physiol. 1869. S. 174 u. 521.

3) Centralbl. f. d. med. Wiss. 1876, No. 15. Dieses Archiv Bd. LXvill. 
legen mag, dieselben jedenfalls in unseren Fällen ihre Wirkung durch das vasomotorische Centrum kundgaben. Eine Bekräftigung des eben Gesagten können wir auch in der schon mehrmals erwähnten Graves'schen Krankheit erblicken.

Obgleich die Frage über das Wesen derselben noch lange nicht abgeschlossen ist, so sind doch die meisten Autoren, wenn sie auch in Einzelheiten von einander abweichen, darin einig, dass jener Krankheit eine Affection des Sympathicus zu Grunde liegt. Trousseau (l. c. T. III. p. 583) sagt gerade heraus, dass er in derselben zum mindesten eine Neurose erblickt, wenn nicht eine materielle Veränderung in den Ganglien, und führt dafür unter anderen Beweisgründen das Fieber an und die sogenannten taches cérébrales.

Friedreich ${ }^{1}$ ) legt den ganzen Prozess Veränderungen in dem Gangliensystem zur Last und namentlich einer Paralyse der Vasomotoren. Eulenburg ${ }^{2}$ ) leitet den Prozess gleichfalls von einer Affection des Sympathicus ab, lässt aber die Frage über die genauere Localisation - ob im Hals-, ob im centralen Theile offen. Peter ${ }^{3}$ ) sieht in der Graves'schen Krankheit eine Affection des Sympathicus.

Uns liegt die Absicht fern, hier näher auf die einzelnen Anschaungen und Theorien einzugehen, die sich in Betreff der Graves'schen Krankheit geltend machen; wenn wir hier einige Autoren angeführt haben, so geschah das nur, um zu beweisen, dass die Ansicht Trousseau's noch immer den ersten Platz beanspruchen darf. Es ist ja wahr, dass in nenester Zeit (x. S $e^{4}{ }^{4}$ ) als ein lebhafter Gegner der herrschenden Meinung aufgetreten ist und sowohl alle Herzneurosen, als die Gravessche Krankheit ausschliesslich vom $\mathrm{N}$. vagus abhängig gemacht wissen will. Aber gerade diese Ausschliesslichkeit bringt es mit sich, dass seine Beweisgründe nicht immer der Kritik Stich halten und sich häufig widersprechen.

Die Graves'sche Krankheit beruht also, der Mehrzahl der Autoren nach, auf einer Neurose des Sympathicus, und von diesem

1) Lehrbuch der Herzkrankbeiten. 1867.

2) Liaussen's Handbuch. Theil VII. S. 89. 1875.

3) Traité clin. et prac. des maladies du coeur. Paris 1883. p. 743.

4) Du diagn. et du trait. des maladies du coeur. Paris 1883. 
Gesichtspunkte aus erklärt sich auch - allerdings nicht ohne einige Schwierigkeiten - das gemeinsame Auftreten der 3 dieser Form eigenthümlichen Symptome.

Aber abgesehen von diesen 3 Cardinalsymptomen giebt es bei der Graves'schen Krankheit noch eine ganze Reihe secundärer Erscheinungen, die, wenn sie auch keine "conditio sine qua non" bilden, doch oft als Begleiterscheinungen auftreten. Wir meinen hier namentlich Fieber, Schweisse, Speichelabfluss, Durchfälle und Husten. Weiter oben haben wir bereits von diesen Symptomen gesprochen und sie zu deuten versucht. Hier berühren wir blos die Frage vom Fieber, welche für uns von vorwiegendem Interesse ist. Obgleich das Fieber kein Cardinalsymptom der Graves'schen Krankheit bildet, so begegnen wir ihm doch in der weitaus grössten Zahl der Fälle, ja nach Eulenburg soll es sogar bei genauerer und häufigerer Beobachtung in jedem einzelnen Falle zu finden sein.

Wie soll man sich nun dieses Fieber erklären? Trousseau beruft sich bei der Erwähnung desselben in der Graves'schen Krankheit darauf, dass auch bei einigen anderen Sympathicuserkrankungen eine T'emperatursteigerung beobachtet wird. Ferner weist er darauf hin, dass auch bei verschiedenen Nervenkrankheiten, z. B. bei der Zuckerharnruhr, oft ein fieberhafter Zustand eintritt. Indem er nun die Pulsbeschleunigung, die Temperaturerhöhung und die taches cérébrales zusammenfasst, sieht er sie als Folgezustände einer und derselben Ursache an, nehmlich einer tief greifenden Veränderung im Sympathicus und namentlich im vasomotorischen System. Eulenburg und Peter treten gleichfalls für eine Entstehung des Fiebers auf vasomotorischem Wege ein. G. Sée kann, trotz seiner scharfen Opposition gegen die herrschende Meinung mit seiner Vagustheorie das Fieber nicht erklären und erkennt es als ein vasomotorisches Phänomen an.

So ist denn kein Grund vorhanden, den vasomotorischen Charakter des Fiebers bei der Graves'schen Krankheit zu leugnen.

Nun giebt es aber noch ein Factum, das als Beweis für eine durch veränderte Thätigkeit der Vasomotoren mögliche Temperatursteigerung dienen kann.

Während wir nehmlich in Fällen von Darmneurosen die 
Herzthätigkeit und die Temperatur verfolgten, fanden wir, dass meist, wenigstens in starkeu Anfällen von Darmtetanus, eine Temperatursteigerung und eine Vergrösserung des queren Herzdurchmessers als Begleiterscheinungen auftraten.

Auf diesen Umstand haben wir bereits in unserer Abhandlung über die Neurosen des Darmes aufmerksam gemacht, doch nur oberflächlich, da wir damals nur über eine kleine Casuistik verfügten. Seit der Zeit haben wir uns bereits mehr als einmal von dem eben Gesagten überzeugen können. Schon bei der Besprechung der Darmerscheinungen im Falle II wiesen wir darauf hin, wie sich dieselben vom Standpunkte der Basch'schen Versuche aus erklären lassen. Wenn aber, wie Basch gezeigt hat, diesen Darmerscheinungen vasomotorische Eigenheiten des $\mathrm{N}$. splanchnicus zu Grunde liegen, so müssen augenscheinlich die Temperatursteigerungen und die Vergrösserung des Herzdurchmessers, welche im Gefolge des Darmtetanus einhergehen, ebenfalls vasomotorischen Einflüssen zugeschrieben werden. Da wir die vorwiegend vasomotorische Bedeutung der N. splanchnici kennen, so müssen wir diese Schlussfolgerung als eine gesetzmässige ansehen. Also auch hier verdankt die Temperaturerhöhung ibre Entstehung ausschliesslich einer Sympathicusreizung.

Alle diese Momente können uns in unsrer bereits oben ansgesprochenen Meinung, dass nehmlich unseren Fällen eine thermische Neurose des vasomotorischen Centrums zu Grunde liege, nur noch mehr bestärken.

Nachschrift. Nach Beendigung dieses Aufsatzes kam mir das neue klassische Werk von Prof. v. Recklinghausen (Handbuch der allgemeinen Pathologie des Kreislaufes und der Ernährung 1883.) zu Gesicht. In ihm fand ich folgende Citate, die meine Auseinandersetzungen, fast möchte ich sagen bestätigen, mindestens aber ihnen ein Bürgerrecht verleihen.

S. 506. „Ob auch den sog. essentiellen Fiebern allgemein eine Entstehung aus einer Blutveränderung vindicirt werden darf, darüber lässt sich gegenwärtịg noch kein Urtheil fällen. Es bleibt vielmehr die Möglichkeit offen, dass unter diesen Fiebern manche sind, bei welchen die erste Veränderung im centralen Nervensystem Platz greift. Wenn ein heftiger Schreck einen 
Fieberanfall inducirt, wie es beobachtet wurde, wenn epileptische Anfälle einen Zustand der Erregung mit Temperatursteigerung herbeiführen, wenn die epileptiformen Anfülle, die im Verlaufe der allgemeinen Paralyse auftreten, mit einer bedeutenden Temperatursteigerung einhergehen (Clouston), wenn bei Geisteskranken periodisch Agitationszustände sich einstellen, welche regelmässig auch Fiebertemperaturen veraulassen (Westphal), so müssen wir anerkennen, dass die Fieberquelle ihren Sitz auch im centralen Nervensystem haben kann - ganz in Uebereinstimmung damit, dass wir den Nerven einen Einfluss auf den Stoffwechsel der Gewebe nicht nur zur Zeit der evidenten Functionirung, sondern auch während der Ruhe zuerkennen."

S. 507. „Sollen wir nun nach dem heutigen Stande unserer Erkenntniss das Fieber definiren, so können wir dasselbe als eine Störung bezeichnen, in welcher die Umsetzung der lebenden Materie des Körpers, in hervorragender Weise diejenige der eiweissreichen Substanzen der Gewebe, des sog. lebendigen Eiweiss (Pflüger), gesteigert ist, ohne dass die Art dieses chemischen Stoffwandels qualitativ anders geworden wäre wie normal. Diese Steigerung kann vom Nervensystem, aber wahrscheinlich auch vom Blut aus hervorgerufen werden. Unter den mannichfaltigen Apparaten des Körpers, welche fieberhaft erregt werden, steht der motorische Apparat des Gefässsystems, das Herz und die Gefässmusculatur, obenan, beherrscht von dem vasomotorischen Nervensystem, welchem daher in der Fieberbewegung eine Hauptrolle zufällt."

\section{IX.}

\section{Ueber Actinomyces.}

Berichtigung zu einem Referate des Herrn Prof. Johne in Dresden.

Von Dr. Oscar Israel, Assistenten am pathologischen Institute zu Berlin.

In No. 4 der "Fortsehritte der Medicin" vom 15. Februar d. J. referirt Herr Johne über meine Arbeit "Ueber die Cultivirbarkeit der Actinomyces“ (dies. Archiv Bd. 95. S. 140).

Nachdem der Redacteur der "Fortschritte", Herr Friedländer, einer Erwiderung meinerseits wegen Platzmangels die A ufnahmeversagt 